\title{
Democratic Republic of the Congo: Staff-Monitored Program
}

This paper on the Staff-Monitored Program for the Democratic Republic of the Congo was prepared by a staff team of the International Monetary Fund as background documentation for the periodic consultation with the member country. It is based on the information available at the time it was completed on June 29, 2006. The views expressed in this document are those of the staff team and do not necessarily reflect the views of the government of the Democratic Republic of the Congo or the Executive Board of the IMF.

The policy of publication of staff reports and other documents by the IMF allows for the deletion of market-sensitive information.

To assist the IMF in evaluating the publication policy, reader comments are invited and may be sent by e-mail to publicationpolicy@imf.org.

Copies of this report are available to the public from

International Monetary Fund $\bullet$ Publication Services

700 19th Street, N.W. • Washington, D.C. 20431

Telephone: (202) 6237430 • Telefax: (202) 6237201

E-mail: publications@imf.org • Internet: http://www.imf.org

Price: $\$ 15.00$ a copy

\section{International Monetary Fund Washington, D.C.}





\title{
INTERNATIONAL MONETARY FUND
}

\section{DEMOCRATIC REPUBLIC OF THE CONGO}

\section{Staff-Monitored Program}

\author{
Prepared by the African Department \\ (In consultation with other departments)
}

Approved by Robert Corker and Anthony Boote

June 29, 2006

- The authorities are requesting a staff-monitored program (SMP) for April 1December 31, 2006. The main objectives of the SMP are to preserve macroeconomic stability during the elections and give the authorities the opportunity to establish a track record of policy implementation, which would pave the way for a successor Poverty Reduction and Growth Facility (PRGF) arrangement.

- The PRGF arrangement with the Democratic Republic of the Congo expired on March 31, 2006, before the last review was completed, because of fiscal slippages and delays in implementing structural measures. One quantitative performance criterion and four structural performance criteria were not met at end-September 2005. The arrangement, approved on June 12, 2002, in the amount of SDR 580 million (108.8 percent of quota), was extended to March 31, 2006, at the time of the fifth review on August 27, 2005. Disbursements totaled SDR 553.5 million.

- The authorities have implemented the seven prior actions shown in Appendix 1, Table 2.

- Discussions on the program took place in Washington during the Spring meetings in April 2006 and in Kinshasa May 25-June 1. The staff team comprised Messrs. Briançon (head), Ben Ltaifa, Hartley (all AFR), Feler (PDR), Williams (FAD), and Maret (Resident Representative).

- The team met with the Vice President for Economic and Financial Affairs; the Ministers of Finance, Budget, Social Affairs, Economy, Commerce, Civil Service, Planning, Transportation, Mining, Energy, Education, and Health; the Governor of the Central Bank; other senior officials; and donors.

- With final PRSP expected to be adopted toward mid-2006, the DRC could reach the completion point under the enhanced Heavily Indebted Poor Countries (HIPC) Initiative and benefit from debt relief under the Multilateral Debt Reduction Initiative (MDRI) in the second half of 2007, provided that the following conditions are met: satisfactory implementation of the SMP; completion of the first review of a new PRGF arrangement covering at least a six-month period; implementation for one year of the poverty reduction strategy; and implementation of completion point triggers. Total debt relief would amount to more than US\$7 billion in net present value terms.

- The Technical Memorandum of Understanding, and Appendices on Fund Relations and World Bank Relations for the SMP will be made available on the IMF website. 
Executive Summary

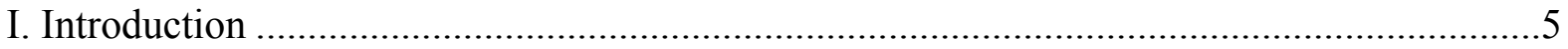

II. Economic developments in 2005 and Performance Under the PRGF ...............................5

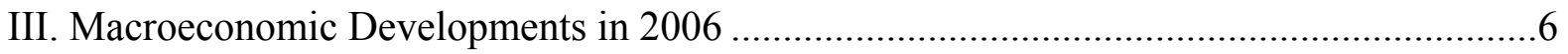

IV. The program for April-December 2006................................................................

A. Macroeconomic Objectives and Policies ........................................................ 9

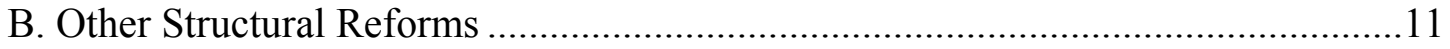

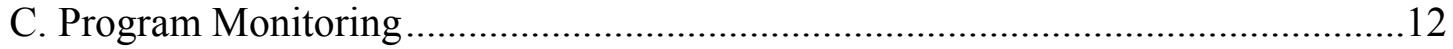

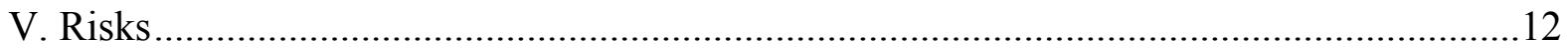

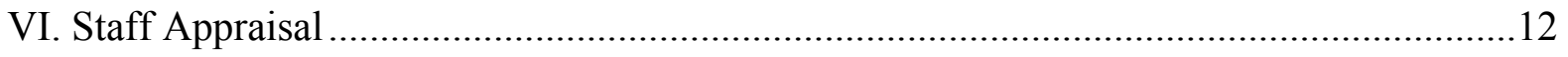

\section{Boxes}

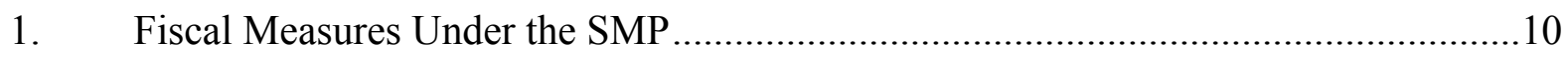

Figures

1. Macroeconomic Developments, 2002-05 ......................................................... 7

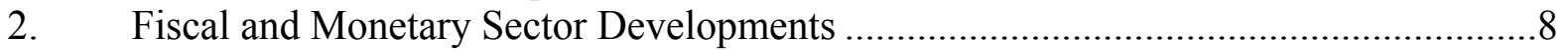

\section{Tables}

1. Selected Economic and Financial Indicators 2004-06 ........................................15

2. Central Government Financial Operations, 2004-06 ...........................................16

3. Summary of Central Government Operations, 2004-06 ....................................... 17

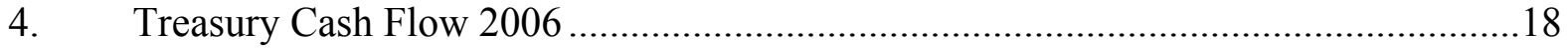

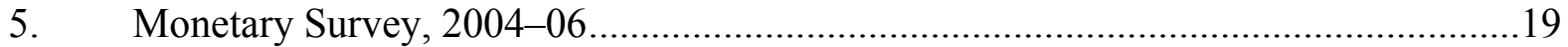

6. Accounts of the Central Bank of the Congo, 2004-06 .........................................20

7. Balance of Payments, Summary 2004-09 .........................................................21 
Appendices

I. Letter of Intent

Attachment I. Memorandum of Economic and Financial Policies for

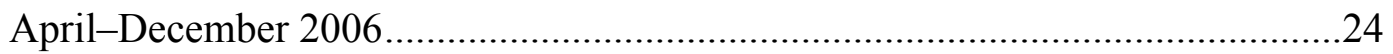

Attachment II. Technical Memorandum of Understanding ...........................................35

Annex I. Definition of External Debt ………………..................................43

Annex II. Definitions of Poverty Reducing Spending ..................................45

Annex III. Budget Tracking Statements ........................................................48

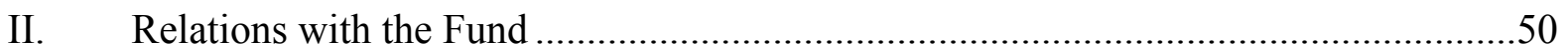

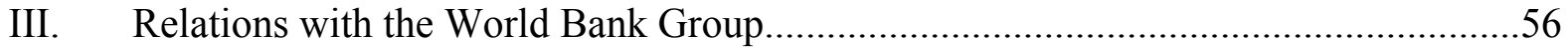

Appendix Tables

1. Performance Criteria and Structural Benchmarks, 2005 ..........................................30

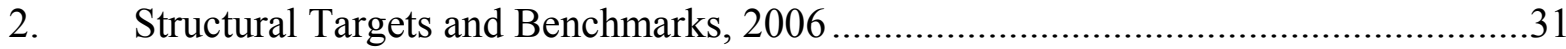

3. Summary of Action Plan for the Central Bank of the Congo (BCC), 2006 .................32

4. Quarterly Quantitative Performance Criteria and Benchmarks, 2005 ..........................33

5. Quarterly Quantitative Benchmarks, 2005-06 .........................................................34 


\section{Executive Summary}

The DRC was successful in restoring macroeconomic balances under the PRGFsupported program. Real GDP growth turned positive, reaching 6.5 percent in 2005 , inflation fell sharply, and the exchange rate became more stable.

The last review under the PRGF arrangement, however, could not be concluded before the arrangement expired at end-March 2006 because of fiscal slippages and delays in structural reforms.

For the first time in $\mathbf{4 0}$ years, the DRC will hold general elections to select its leader. This will bring an end to the political transition. The first round of presidential and parliamentary elections is scheduled for July 30, 2006. A new government may not be formed before November 2006 if two rounds of voting are required to elect the President.

The SMP is intended to preserve macroeconomic stability during the protracted elections and give the authorities the opportunity to establish a strong track record of policy implementation. In the absence of confirmed external budget support for the rest of the year, the program's main goal is to keep government spending in line with revenue so that bank financing will not be needed. This will require a sharp tightening of fiscal policy, to be achieved by improving tax collection and adhering to a monthly cash-flow plan. The central bank will also need to maintain a tight monetary policy to contain inflation, keep the value of the currency stable, and increase the low level of international reserves.

The SMP includes measures for improving public resource management and strengthening central bank operations. At the fiscal level, the objectives are to strengthen tax collection, enhance budget execution and monitoring, and improve the transparency of government operations. At the central bank, the reforms seek to enhance its ability to conduct monetary policy by strengthening key departments and monetary policy instruments.

However, the staff is concerned about the takeover of an ailing state-owned bank as the fiscal cost and the financing of this operation have not yet been determined.

Implementation of the SMP faces major risks. As the elections approach, political and security tensions are likely to rise, diverting attention from the program. The authorities may also come under increasing pressures to ease fiscal and monetary policies and in particular to increase spending. To compound this, the absence of external budget support increases-the risk that the authorities will not be able to service all external debt and that an exogenous shock could lead to a rapid depreciation of the currency and high inflation. 


\section{INTRODUCTION}

1. In the attached Letter of Intent dated June 29, 2006 (Appendix I), the Congolese authorities request a staff-monitored program (SMP) for April 1-December 31, 2006, in support of the economic program they spell out in the accompanying Memorandum on Economic and Financial Policies (MEFP).

2. The main objective of the SMP is to preserve macroeconomic stability through the upcoming elections. The SMP seeks to keep government spending within government resources so as to avoid recourse to domestic bank credit to finance the deficit, which in the past was the main source of macroeconomic instability. The authorities believe that an SMP would facilitate their dialogue with development partners and continue their close engagement with the Fund.

3. Political tensions are high in the run-up to the first round of the presidential and parliamentary elections scheduled for July 30, 2006. Given the time required for organizing two rounds of voting, if necessary, and for the Supreme Court to confirm the results, a new government may not be formed before November. The new constitution specifies that transition institutions should remain in place until the new government is formed, but some political parties are questioning their legitimacy after June 30, when, according to the Sun City Accord, the political transition was to end. To help secure the elections, the UN Security Council has extended the mandate of its troops (MONUC) through September 2006 and the European Union has accepted a UN request to send troops.

\section{ECONOMiC DEVElopments In 2005 And PERformance UNDER THE PRGF}

4. Economic developments were mixed in 2005. Real GDP growth held steady at 6.5 percent, sustained by the rebuilding of the country. After peaking at 27 percent in August, 12 -month inflation receded gradually to 21.3 percent at year end, helped by a 15 percent appreciation of the Congo franc in the second half of the year. However, gross international reserves declined to the equivalent of 2.6 weeks of imports, mainly because a rising share of government spending was paid for in US dollars so as to keep money growth in line with the program target.

5. Large fiscal slippages in the second half of 2005 led to an increase in bank financing of the budget. Overruns in current spending amounting to 2.5 percent of GDP were due to spending by political institutions and the military and to increases in wages granted last fall to ease social tensions. On a more positive note, rising revenue from oil production boosted total government revenue above the anticipated level by 0.9 percent of GDP, limiting the underlying fiscal deficit to 0.2 percent of GDP ${ }^{1}$ However, the outcome was a significant deviation from the planned surplus of 1.6 percent of GDP. The overall

\footnotetext{
${ }^{1}$ The underlying fiscal balance excludes grants, foreign financed investment, exceptional spending, external interest payments, and arrears.
} 
deficit was only slightly larger than targeted because of delays in foreign-financed spending on capital projects and the preparation for the elections.

6. The pace of structural reforms was slow. It became increasingly difficult to reach consensus on measures while the transition government was concerned with adoption of the new Constitution and other laws required for the elections. Nevertheless, on December 30, 2005, the President signed the decree designating the customs office (OFIDA) as the sole government agency to operate the one-stop window at the port of Matadi and to value imports. With technical assistance from the Fund, the central bank also made progress in strengthening the operations of key departments.

\section{Corruption remained a major problem, particularly in natural resource} management. However, the authorities signed the Extractive Industries Transparency Initiative (EITI) in March 2005 and created a multi-stakeholder National EITI Committee in November.

8. Fiscal slippages and slow progress in structural reforms prevented conclusion of the last review of the PRGF arrangement, which expired on March 31, 2006. The performance criterion on net credit to the government and the four structural performance criteria for September 2005 were not met (see Appendix I, Tables 1 and 4). In addition, with high government spending continuing, three quantitative indicators for December were not met.

\section{Macroeconomic DeVelopments in 2006}

9. The macroeconomic environment is still very fragile. The increase in economic activity appears slightly less buoyant than expected because the private sector is becoming more cautious as the elections approach. Twelve-month inflation declined to less than 6 percent in April, but rose again in May when the consumer price index jumped by 2 percent. The uptick in inflation is the consequence of a surge in money-financed government spending in April that was followed by a 2 percent depreciation in the Congo franc against the US dollar in early May. The Central Bank responded to the increase in bank liquidity by selling foreign exchange and raising its key interest rate by 3 percentage points, to 28.5 percent, at end-May. Nevertheless, gross international reserves increased to 3.0 weeks of nonaid-related imports. Preliminary data indicate that government spending remained higher than anticipated in May; but the authorities did not have recourse to domestic bank financing because of windfall gains in oil revenue.

10. The authorities have made some progress on structural measures. In particular, to enhance transparency in government operations, they have posted on the Internet summaries of government monthly spending and cash flow plans and outcomes for March and April. Double-entry accounts for the Treasury have been updated through December 2005, and the authorities are completing discussions on draft ministerial decrees to improve collection of government revenue (Appendix I, Table 2). 


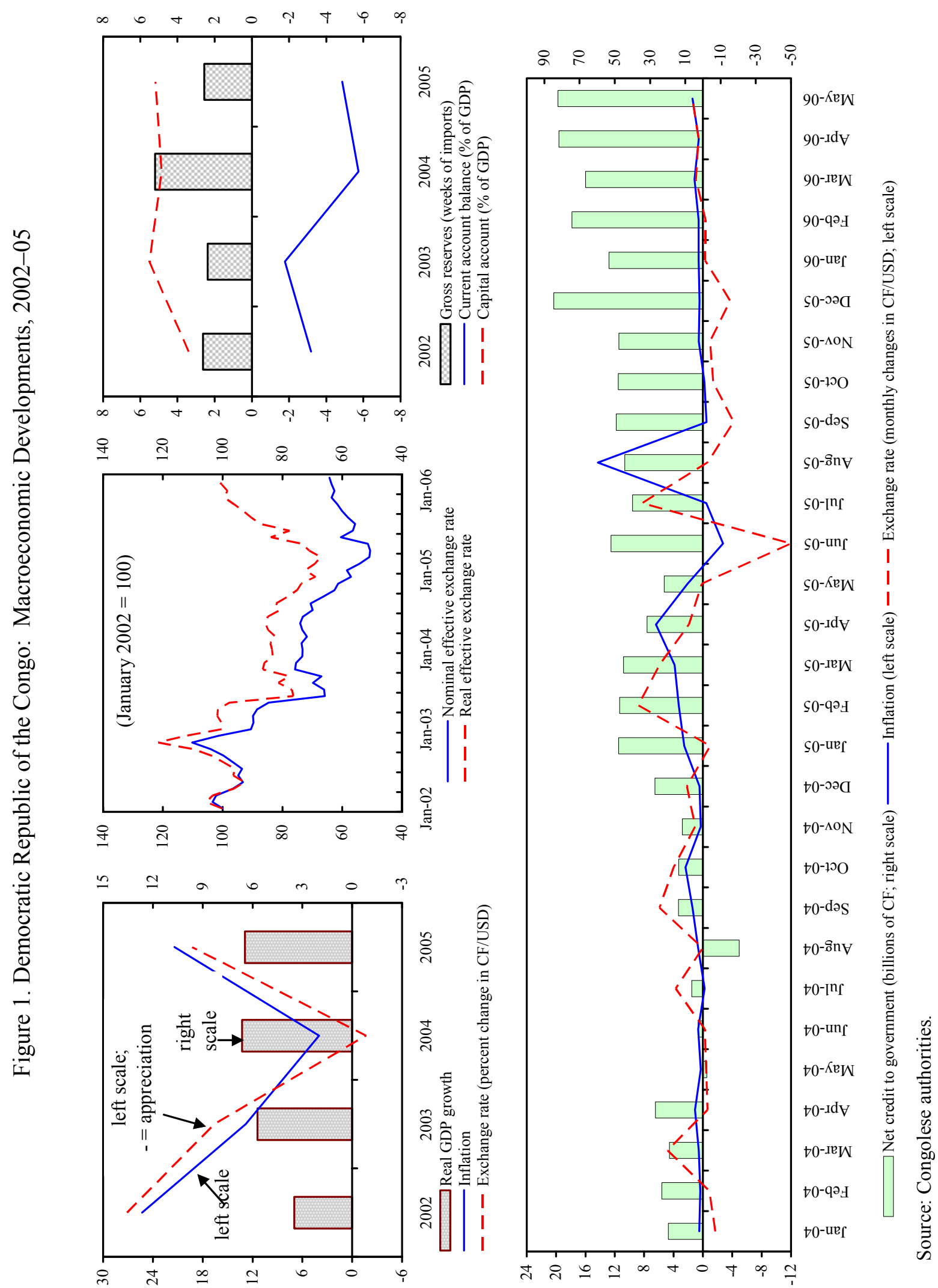


Figure 2. Democratic Republic of the Congo: Fiscal and Monetary Sector Developments

Fiscal Sector, 2000-05

(In percent of GDP)
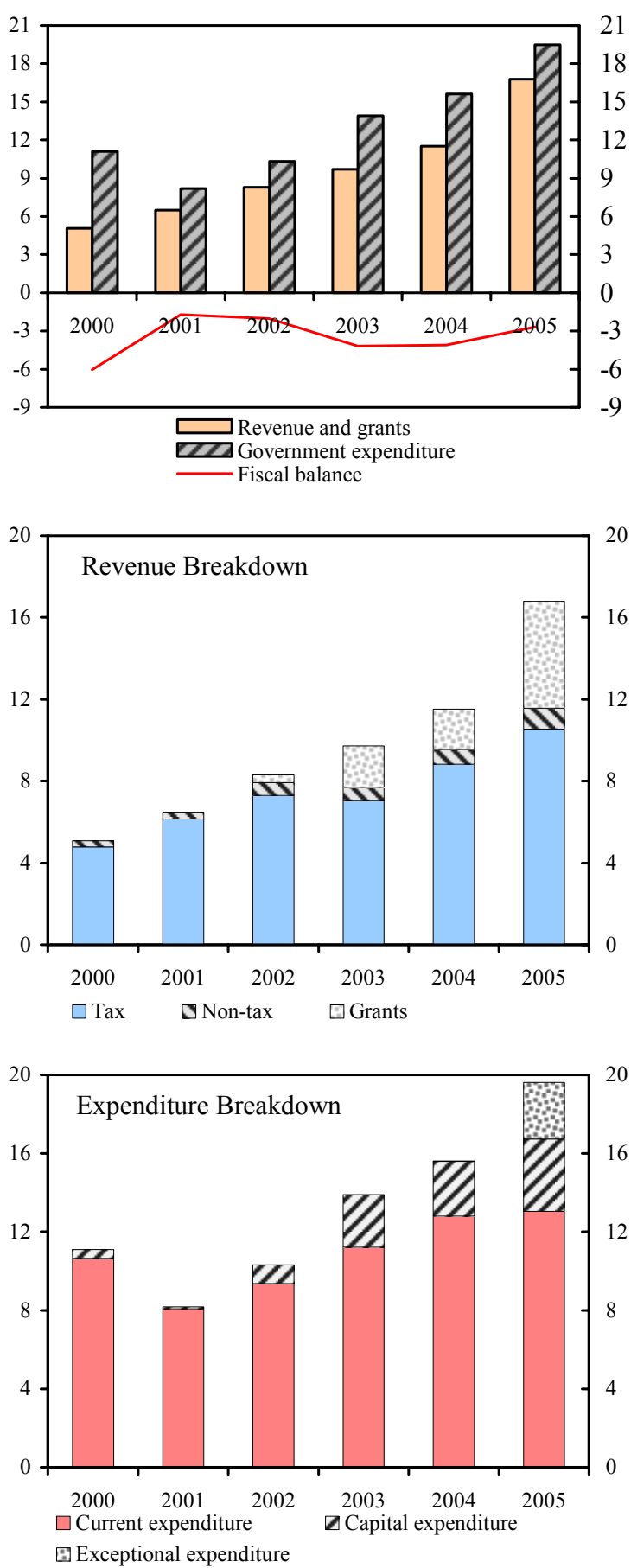

Monetary Sector, 2001-05

(In billions of Congolese francs)
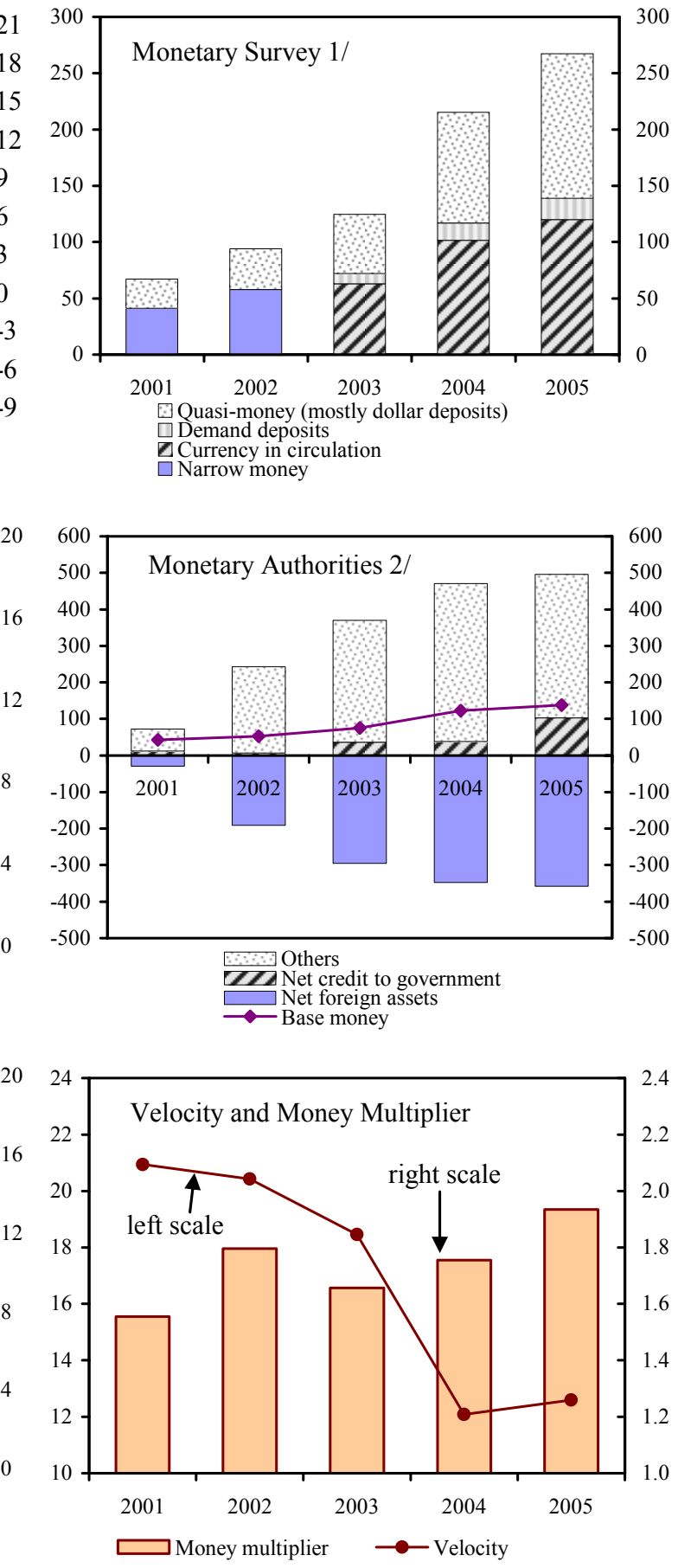

Source: Congolese authorities and IFS.

1/ 2001-02 data from IFS; 2003-05 data from authorities, based on current exchange rates.

2/ All data from authorities; 2001-02 data is based on program exchange rates; 2003-05 data is based on current exchange rates. 


\section{THE PROGRAM FOR APRIL-DECEMBER 2006}

11. The economic policy challenge is to maintain macroeconomic stability without external budget support during an election year. The authorities are very aware that market confidence in government policy is low and any increase in bank-financed government spending would almost immediately translate into a depreciation of the Congo franc and increased inflation. Therefore, they recognize the importance of keeping fiscal policy tight for the rest of this year. The SMP also includes structural measures to improve public resource management and transparency.

\section{A. Macroeconomic Objectives and Policies}

12. The macroeconomic objectives for $\mathbf{2 0 0 6}$ are ambitious. Real GDP growth is projected at 6.5 percent and 12-month inflation is expected to be held below 10 percent. Increasing gross international reserves to about 4 weeks of imports will also be essential. While such a level is still very low, it may require central bank intervention on the foreign exchange market because no external budget support has been secured for the rest of 2006 . The authorities are seeking to mobilize such support, which is crucial to maintaining macroeconomic stability.

13. The government recognizes the importance of tightening the fiscal stance by raising revenue through more forceful tax administration and reining in spending (Box 1). It will seek to achieve an underlying fiscal surplus equivalent to $2 \frac{1}{4}$ percent of GDP in 2006, compared with a deficit of 0.2 percent of GDP recorded in 2005. At the same time, the overall fiscal deficit, which includes foreign-financed spending for capital projects, the political transition process, and restructuring the economy, would be cut by one half to $1 \frac{1}{2}$ percent of GDP in 2006.

14. Government revenue is projected to rise by 23 percent, 0.9 percentage points of GDP, in 2006. The projection assumes that tax collection will weaken during the elections following an increase of 40 percent in non-oil revenue in the first five months of 2006 over the same period in 2005. Meanwhile, the contribution to government revenue from oil production is expected to rise from 2.9 percent of GDP in 2005 to 3.2 percent in 2006, in line with the increase in the international price of crude oil.

15. Given the lack of external budget support, government spending will be kept at 23.2 percent of GDP, or 1.2 percentage points below the level voted with the 2006 budget. The wage bill is to rise by 0.3 percentage points of GDP to take into account the increase in benefits granted last fall. Keeping the wage bill below its ceiling will, however, require removing ghost employees identified in the civil service and military censuses and putting into operation a new payroll system by August 2006. Control over the military payroll will be strengthened with the assistance of experts from the European Union. The authorities said that they will keep social spending close to its budgeted level. Whether this will be possible depends on both the available revenues and on the authorities' capacity to contain outlays for political institutions and the military, where pressures to spend are high on the eve of the elections. 


\section{Box 1. Fiscal Measures Under the SMP}

Strengthening public resource management is crucial to the success of the SMP.

\section{Revenue measures:}

- Implement the presidential decree and the manual of customs procedures that make the customs office the sole tax collection agency in Matadi.

- Sign the ministerial resolution that would facilitate implementation of the pre-inspection contract between the customs agency and a private firm.

- Adopt unique taxpayer numbers that would allow tax collection agencies to mitigate fraud and enforce greater compliance.

- Open two tax collection centers in the provinces targeting small to medium-sized enterprises.

- Sign interministerial decrees transferring to the nontax agency DGRAD the collection of all fees and duties currently collected by autonomous agencies.

- Suspend the granting of any new exemptions and for the duration of the SMP limit existing ones to those already granted under the investment, mining, and forestry codes.

- Raise base prices used for calculating the excise tax on oil products to world market prices to achieve revenue objective.

\section{Expenditure measures:}

- Keep the wage bill under CF180 billion by implementing the results of the civil service and military censuses and simplifying the payroll system.

- Observe the monthly spending plan for each ministry and enforce ceilings by incorporating it into the computerized expenditure procedures.

- Approve budgetary advances only after ministries have justified their use of previous advances.

- Implement a double-entry accounting system to enhance the transparency of public sector operations.

- Audit the use of HIPC savings from 2003 through 2005.

16. To achieve its fiscal objectives, the government has prepared monthly cash flow and spending plans for each ministry. Monthly updates to the plans will ensure that they stay consistent with monetary objectives. Spending plans are integrated into the computerized expenditure procedures to make it easier to enforce spending limits; similarly, posting the spending plans and actual spending on the Internet should make it easier to monitor budget execution.

\section{Notwithstanding the fiscal effort, there remains a financing gap in the fourth} quarter of 2006 that is estimated at 0.8 percent of GDP (US\$70 million). The authorities are seeking external budget support to fill the gap. Without such assistance, the government may have difficulties paying its debt service, especially since interim assistance under the 
enhanced HIPC Initiative has been interrupted. The staff will assess the budget position and discuss any adjustment needed with the authorities during the first review of the SMP. It may not be possible to increase government spending, reduce net credit to the government, and increase international reserves as currently intended. Staff also urged the authorities to save some windfall gains from oil revenue.

18. Monetary policy will continue to be directed at containing inflation in the context of a flexible exchange rate system. The Central Bank of the Congo (BCC) intends to keep monetary policy tight by containing the growth of net domestic assets while gradually building up international reserves. The conduct of monetary policy may be hampered by weak policy instruments and limited market confidence in the ability of the central bank to offset expansionary fiscal policy and control base money growth. This largely explains the difficulties the BCC has had in selling treasury bills to mop up excess liquidity.

\section{To improve the conduct of monetary policy, the $\mathrm{BCC}$ recognizes it must} strengthen its operations, in particular liquidity management and forecasting. As Fund technical assistance has recommended, it is essential to restructure the departments of the central bank that are in charge of currency issues, accounting, internal audit, and banking supervision. The $\mathrm{BCC}$ should also recruit an international firm to audit its organization so that it can reduce its operating losses before it is recapitalized. The BCC recently transferred CGF 532 billion (US\$1.2 billion) in accumulated losses to the government's account. The terms of the transfer and its budgetary impact will be specified for the 2007 budget.

20. The central bank needs to ensure that the announced takeover of the Union des Banques Congolaises (UBC) by the Banque Congolaise (BC) preserves the integrity of the banking system at minimal cost to the budget. Liquidation of UBC, a majority stateowned bank, was originally specified in the plan for restructuring the Congolese banking system adopted in 2002. Although UBC is virtually bankrupt, the government decided to move ahead with the BC takeover of the UBC because of its strategic importance and wide branch network. Fund and World Bank staffs had urged the authorities to postpone the transaction and take time to (i) determine its fiscal cost - currently estimated at US\$13 million to cover UBC's losses and US\$2.7 million for employees severance package - and its financing, including from the central bank; (ii) assess possible contingent liabilities; and (iii) conduct due diligence on the shareholders of the $\mathrm{BC}$ and their capacity to manage the bank and increase its capital. The authorities have agreed to have an accounting firm audit the two banks, and if necessary, to discuss with the BC ways to finance the UBC's losses in excess of US\$13 million so that the cost is not borne by the government. The authorities will also discuss with Fund and World Bank staffs the results of the audits and their implications for the 2007 budget and the banking system.

\section{B. Other Structural Reforms}

21. The ability to implement structural reforms during the election period will be limited. Besides the reforms already discussed, the SMP mostly covers measures that started under the last PRGF-supported program. 
22. A special effort will be made to improve governance. The authorities will place a moratorium on (i) new tax exemptions, including under the investment, mining, and forestry codes until the end of 2006; and (ii) joint ventures between public entities and private companies.

\section{Program Monitoring}

23. The staff will monitor the program through a set of quantitative and structural benchmarks (MEFP, Tables 2 and 5). End-June and end-September 2006 are test dates for the quantitative targets.

\section{RISKS}

24. The months ahead are likely to be marked by periods of political and security tensions as the country enters a protracted electoral period, during which some may question the legitimacy of transitional institutions. Focus on the elections can be expected, in general, to distract the government from the strong determination required to consolidate macroeconomic stability. Demands for additional public expenditure are likely to put considerable pressure on the fiscal targets under the SMP. In particular, containing spending by political institutions and the military may be especially difficult in the coming months, while trade unions are likely to call for higher wages.

25. Failure to implement the required tight fiscal policy would exacerbate macroeconomic risks. It could lead to a rapid depreciation of the currency and a pick up in inflation, which would take time to correct. The central bank would have difficulties increasing international reserves, and thereby reduce further its ability to mitigate the impact of exogenous shocks.

26. The government may also have difficulties meeting all its external debt obligations in the absence of external budget support. While the authorities are expected to continue meeting the DRC's debt service obligations to the Fund and other multilateral institutions, they will have difficulties meeting all its debt obligations to bilateral creditors, in particular on the capitalized moratorium interests to Paris Club creditors that fall due at endof September (more than US\$40 million).

\section{Staff Appraisal}

27. Progress toward macroeconomic stability has been remarkable since the PRGFsupported program was approved in June 2002. After a long period of civil and military strife, the transition government implemented coherent economic and structural policies. The result is strong economic growth, an improvement in per capita income, a drop in inflation, and a relatively stable exchange rate. The upcoming elections, which will offer the population its first opportunity in more than forty years to select national and provincial leaders, will bring the political transition to an end. Rapid formation of a government will be important for implementing the poverty reduction strategy and improving the investment climate. 
28. It is regrettable that policy implementation has weakened since mid-2005, precluding completion of the sixth and last review of the PRGF arrangement. Failure to rebuild foreign exchange reserves has left DRC highly vulnerable to exogenous shocks and with limited fiscal options.

29. The purpose of the SMP is to maintain the stability of the economy through the elections. Success under the SMP would set the stage for discussions on a successor PRGF arrangement once a new government is in place.

30. For the SMP to be successful, the authorities should avoid having recourse to bank financing to cover the fiscal deficit. Hence, the government needs to limit cash spending to available resources by improving revenue collection and adhering to its monthly cash flow and spending plans. In particular, it needs to limit current outlays, which in the past have been a source of slippage. The staff urges the authorities to complete the civil service census and strengthen payroll management so that the ceiling on the wage bill can be enforced.

31. The staff urges the BCC to maintain a tight monetary policy to contain inflation and preserve exchange rate stability. At the same time, international reserves need to be shored up lest the central bank's limited ability to mitigate the impact of exogenous shocks be further compromised. To enhance the effectiveness and the credibility of monetary policy, however, the BCC must improve its monetary policy instruments, international reserve management, and the transparency of its operations, especially its accounts, by implementing recommendations from Fund technical assistance. The BCC should also conduct an organizational audit to reduce its structural deficit before recapitalization.

32. The Fund and World Bank staffs are very concerned about the takeover of the Union des Banques Congolaises by the Banque Congolaise. It is crucial for the authorities to assess the fiscal cost and the financing of this operation, and conduct due diligence on the capacity of BC's shareholders and management to lead the new bank and increase its capital.

33. Corruption is deeply ingrained and is a major obstacle to the improvement of both public resource management and the business climate. Among measures in the SMP to improve matters are computerizing and streamlining budget procedures, posting budget execution on the Internet, tracking poverty-related expenditure, and auditing the use of HIPC savings. It is also urgent to strengthen the management of mining, forestry, and other natural resources to ensure that those sectors contribute to growth and government revenue. 
34. The risks to the SMP going forward are considerable. Risks stem not only from political and security tensions during the elections, but also from strong pressures to ease fiscal and monetary policies. Put another way, sustaining ownership of the SMP will be a major challenge.

35. The authorities need nonetheless to realize that a lapse in ownership will have major consequences on macroeconomic stability that would take the new government significant time to repair. Recourse to domestic bank financing of the budget would risk a major weakening of the currency and a new cycle of high inflation.

36. The SMP also has a sizable financing gap during the fourth quarter. Lack of external budget support may make it very difficult for the DRC to keep current on external debt service. However, strong implementation of the SMP during the elections would be more likely to elicit support from external donors. 
Table 1. Democratic Republic of the Congo: Selected Economic and Financial Indicators, 2004-06

\begin{tabular}{|c|c|c|c|c|c|}
\hline & \multirow[t]{2}{*}{2004} & \multicolumn{2}{|l|}{2005} & \multicolumn{2}{|c|}{2006} \\
\hline & & $\begin{array}{l}\text { IMF Country } \\
\text { Report No. } \\
05 / 374\end{array}$ & Prel. & $\begin{array}{c}\text { IMF Country } \\
\text { Report No. } \\
05 / 374\end{array}$ & Proj. \\
\hline & \multicolumn{5}{|c|}{ (Annual percentage change; unless otherwise indicated) } \\
\hline \multicolumn{6}{|l|}{ GDP and prices } \\
\hline Real GDP & 6.6 & 6.6 & 6.5 & 7.0 & 6.5 \\
\hline GDP deflator & 6.1 & 22.5 & 21.5 & 5.5 & 7.9 \\
\hline Consumer prices, end-of-period & 9.2 & 22.6 & 21.3 & 6.2 & 9.5 \\
\hline \multicolumn{6}{|l|}{ External sector } \\
\hline Exports, f.o.b. (U.S. dollar terms) & 35.3 & 12.6 & 13.1 & 0.1 & 15.5 \\
\hline Imports, f.o.b. (U.S. dollar terms) & 37.4 & 19.9 & 9.3 & 5.7 & 26.1 \\
\hline Export volume & 20.1 & 8.8 & -4.2 & 2.4 & 10.0 \\
\hline Import volume & 26.4 & 17.6 & 3.1 & 5.3 & 26.4 \\
\hline Terms of trade & 3.6 & 1.5 & 11.3 & -2.7 & 5.2 \\
\hline Nominal effective exchange rate $1 /$ & -11.7 & $\ldots$ & -17.6 & $\ldots$ & $\ldots$ \\
\hline \multirow[t]{2}{*}{ Real effective exchange rate $1 /$} & -9.9 & $\ldots$ & 2.5 & $\ldots$ & $\ldots$ \\
\hline & \multicolumn{5}{|c|}{$\begin{array}{l}\text { (Annual change in percent of beginning-of-period broad money; } \\
\text { unless otherwise indicated) }\end{array}$} \\
\hline \multicolumn{6}{|l|}{ Money and credit } \\
\hline Broad money & 72.9 & 25.6 & 24.2 & $\ldots$ & 20.8 \\
\hline Net foreign assets & -18.3 & 24.4 & 3.7 & $\ldots$ & 11.8 \\
\hline Net domestic assets & 92.3 & 3.5 & 21.8 & $\ldots$ & 10.5 \\
\hline \multicolumn{6}{|l|}{ Of which: } \\
\hline Net credit to government & -1.9 & -12.3 & 26.6 & $\ldots$ & -5.8 \\
\hline \multirow[t]{2}{*}{ Credit to the private sector (annual percent change) } & 105.5 & 42.9 & 58.1 & $\ldots$ & 15.5 \\
\hline & \multicolumn{5}{|c|}{ (Percent of GDP; unless otherwise indicated ) } \\
\hline \multicolumn{6}{|l|}{ Central government finance } \\
\hline Total government revenue & 9.5 & 10.7 & 11.6 & 11.5 & 12.5 \\
\hline Grants & 2.0 & 9.3 & 5.2 & 7.5 & 9.5 \\
\hline Total government expenditure 2/ & 15.6 & 22.3 & 19.5 & 20.6 & 23.2 \\
\hline Underlying fiscal balance (cash basis) & -0.9 & 1.6 & -0.2 & 1.0 & 2.2 \\
\hline Overall fiscal balance (payment order basis, incl. grants) & -4.1 & -2.4 & -2.7 & -1.7 & -1.2 \\
\hline Overall consolidated fiscal balance (cash basis, incl. grants) $3 /$ & -2.2 & -2.2 & -8.1 & -1.9 & -0.7 \\
\hline \multicolumn{6}{|l|}{ Investment and saving } \\
\hline Gross national saving & 7.1 & 12.6 & 9.3 & 12.3 & 11.9 \\
\hline Government & -2.2 & 0.0 & -2.0 & 3.6 & 2.3 \\
\hline Nongovernment & 9.2 & 12.6 & 11.3 & 8.7 & 9.5 \\
\hline Investment & 12.8 & 17.7 & 14.2 & 20.2 & 16.7 \\
\hline Government 4/ & 2.8 & 7.2 & 3.7 & 9.2 & 6.7 \\
\hline Nongovernment & 10.0 & 10.5 & 10.5 & 11.0 & 10.0 \\
\hline \multicolumn{6}{|l|}{ Balance of payments } \\
\hline Exports of goods and services & 30.3 & 32.3 & 31.6 & 28.8 & 30.5 \\
\hline Imports of goods and services & 39.2 & 45.6 & 39.3 & 42.0 & 42.4 \\
\hline Current account balance, incl. transfers & -5.7 & -5.1 & -4.9 & -7.9 & -4.8 \\
\hline Current account balance, excl. transfers & -12.3 & -16.2 & -10.7 & -15.4 & -14.8 \\
\hline Gross official reserves (end-of-period, millions of U.S. dollars) & 236.2 & 360.2 & 131.2 & 470.2 & 246.8 \\
\hline \multirow[t]{2}{*}{ Gross official reserves (weeks of nonaid-related imports of goods and services) } & 5.2 & 7.2 & 2.6 & 8.5 & 4.2 \\
\hline & \multicolumn{5}{|c|}{ (Millions of U.S. dollars; unless otherwise indicated) } \\
\hline External public debt & & & & & \\
\hline Total stock, including IMF 5/ & 10,643 & 10,735 & 10,822 & 5,041 & 10,813 \\
\hline Net present value (NPV) of debt $6 /$ & 7,348 & 7,630 & 7,624 & 1,608 & 6,125 \\
\hline NPV (percent of exports of goods and services) $6 /$ & 474.7 & 401.4 & 400.6 & 74.7 & 265.4 \\
\hline Scheduled debt service & 163.2 & 144.8 & 154.1 & 218.1 & 251.6 \\
\hline Percent of exports of goods and services & 8.2 & 6.5 & 6.9 & 9.7 & 9.8 \\
\hline Percent of government revenue & 21.7 & 10.5 & 12.9 & 14.8 & 13.6 \\
\hline Exchange rate, Congo franc/U.S. dollar & & & & & \\
\hline Period average & 397.8 & 488.3 & 474.4 & $\ldots$ & $\ldots$ \\
\hline End-of-period & 444.1 & 480.0 & 431.3 & $\ldots$ & $\ldots$ \\
\hline Memorandum items: & & & & & \\
\hline Nominal GDP (billions of Congo francs) & 2,601 & 3,380 & 3,366 & 3,814 & 3,868 \\
\hline
\end{tabular}

Sources: Congolese authorities and IMF staff estimates and projections.

$1 /$ Change in annual average. Minus sign indicates depreciation.

$2 /$ Includes interest due before debt relief and expenditure financed by HIPC resources.

3/ Cash balance after debt relief on interest payments.

4/ From 2003 onward, includes investment financed by resources released under the enhanced HIPC Initiative.

5/ End-of-period debt stock includes accumulated arrears and reflects the arrears clearance arrangements concluded at the HIPC decision point.

The increase in the projected stock-of-debt at end-2006 reflects the postponement of the HIPC completion point from 2006 to 2007.

6/ Estimates and projections based on end-2002 DSA and after HIPC Initiative assistance assuming completion point in 2007, and includes

assistance beyond the terms of the enhanced HIPC Initiative granted by some Paris Club creditors. Exports are on a three-year backward moving

average. 
Table 2. Democratic Republic of the Congo: Central Government Financial Operations, 2004-06 (Billions of Congo francs; unless indicated otherwise)

\begin{tabular}{|c|c|c|c|c|c|}
\hline & \multirow{2}{*}{$\begin{array}{c}2004 \\
\text { Est. }\end{array}$} & \multicolumn{2}{|c|}{2005} & \multicolumn{2}{|c|}{2006} \\
\hline & & $\begin{array}{c}\text { IMF Country } \\
\text { Report No. } \\
05 / 374\end{array}$ & Est. & $\begin{array}{c}\text { IMF Country } \\
\text { Report No. } \\
05 / 374\end{array}$ & Proj. \\
\hline Total revenue and grants & 299.4 & 674.1 & 564.9 & 721.4 & 850.2 \\
\hline Total revenue & 248.0 & 360.2 & 389.0 & 436.7 & 482.8 \\
\hline Customs and excise & 104.1 & 149.4 & 145.4 & 194.7 & 169.8 \\
\hline Direct and indirect taxes & 71.4 & 97.7 & 111.4 & 135.1 & 141.0 \\
\hline Petroleum (royalties and taxes) & 52.1 & 74.7 & 98.1 & 60.9 & 124.1 \\
\hline Other & 20.4 & 38.4 & 34.1 & 46.0 & 47.9 \\
\hline Total grants & 51.4 & 313.9 & 175.9 & 284.6 & 367.5 \\
\hline Budget grants & 2.4 & 30.3 & 5.6 & 0.0 & 40.5 \\
\hline Project grants & 26.3 & 188.5 & 75.2 & 138.4 & 205.8 \\
\hline HIPC Initiative assistance 1/ & 22.7 & 95.1 & 95.2 & 146.3 & 121.1 \\
\hline Total expenditure & 405.8 & 755.2 & 655.5 & 786.3 & 896.7 \\
\hline Current expenditure & 332.3 & 345.5 & 428.2 & 401.2 & 443.1 \\
\hline Wages 2/ & 93.2 & 124.0 & 146.8 & 152.7 & 180.1 \\
\hline Interest due 3/ & 93.6 & 106.7 & 114.8 & 111.5 & 122.0 \\
\hline Of which: on external debt & 86.1 & 104.7 & 97.1 & 109.5 & 112.0 \\
\hline Transfers and subsidies & 14.8 & 15.9 & 26.9 & 24.1 & 28.2 \\
\hline Other current expenditure & 130.6 & 99.0 & 139.7 & 112.9 & 112.7 \\
\hline Of which: centralized payments & 30.6 & 28.3 & 27.4 & 38.3 & 25.6 \\
\hline Capital expenditure & 72.1 & 233.6 & 113.9 & 319.3 & 240.7 \\
\hline Foreign-financed & 57.0 & 217.3 & 79.5 & 296.3 & 221.0 \\
\hline Domestic-financed & 15.1 & 16.3 & 34.4 & 23.0 & 19.6 \\
\hline Exceptional expenditure 4/ & 0.4 & 157.0 & 97.1 & 0.0 & 178.8 \\
\hline Foreign-financed & 0.4 & 138.8 & 64.2 & 0.0 & 133.0 \\
\hline Domestic-financed & 0.0 & 18.2 & 32.9 & 0.0 & 45.9 \\
\hline Other HIPC-related expenditure 5/ & 1.0 & 19.0 & 21.2 & 65.8 & 34.1 \\
\hline Correction for overpayments & 0.0 & 0.0 & -4.8 & $\ldots$ & ... \\
\hline Change in arrears $6 /$ & -1.4 & 0.0 & -203.8 & -0.1 & -2.1 \\
\hline Float & 11.4 & -15.4 & 2.3 & 0.0 & 0.0 \\
\hline Central bank operational result & -19.1 & -14.0 & -15.1 & -0.4 & -10.0 \\
\hline Overall fiscal balance (cash basis, before interest rescheduling) & -115.4 & -110.4 & -307.3 & -82.9 & -58.5 \\
\hline Underlying fiscal balance $7 /$ & -23.3 & 54.6 & -5.8 & 38.2 & 85.9 \\
\hline Primary fiscal balance (cash basis) & -21.8 & -3.8 & 11.4 & 28.5 & 63.5 \\
\hline Total financing & 89.5 & 110.4 & 307.3 & 82.9 & 58.5 \\
\hline Domestic financing & 0.2 & -26.5 & 261.1 & 0.0 & -29.0 \\
\hline Banking system & 0.2 & -26.5 & 57.4 & 0.0 & -15.494 \\
\hline Non bank & 0.0 & 0.0 & 25.6 & 0.0 & -13.5 \\
\hline Domestic debt relief & 0.0 & 0.0 & 178.1 & 0.0 & 0.0 \\
\hline Foreign financing & 89.3 & 136.9 & 49.1 & 17.1 & 56.1 \\
\hline Amortization due before debt relief & -84.5 & -148.8 & -150.6 & -219.9 & -216.9 \\
\hline Loan disbursement & 89.0 & 196.3 & 115.4 & 158.0 & 148.2 \\
\hline Budget loans & 60.9 & 54.3 & 50.7 & 0.0 & 0.0 \\
\hline Project loans & 28.1 & 142.0 & 64.7 & 158.0 & 148.2 \\
\hline Debt relief before HIPC Initiative assistance & 84.8 & 89.5 & 84.2 & 79.0 & 124.8 \\
\hline \multirow[t]{2}{*}{ Additional financing to be found } & 0.0 & 0.0 & 0.0 & 65.9 & 31.4 \\
\hline & \multicolumn{5}{|c|}{ (Percent of GDP; unless otherwise indicated) } \\
\hline \multicolumn{6}{|l|}{ Memorandum items: } \\
\hline GDP (billions of Congo francs) & 2,601 & 3,380 & 3,366 & 3,814 & 3,868 \\
\hline Current primary expenditure (cash basis) & 9.2 & 7.1 & 15.4 & 7.7 & 8.4 \\
\hline Investment spending (including fraction of HIPC Initiative spending) & 2.8 & 7.2 & 3.7 & 9.2 & 6.7 \\
\hline Underlying fiscal balance (cash basis) $7 /$ & -0.9 & 1.6 & -0.2 & 1.0 & 2.2 \\
\hline
\end{tabular}

Sources: Congolese authorities and IMF staff estimates and projections.

1/ Reflects revised calculation of HIPC Initiative assistance from 2002-based Debt Sustainability Analysis (DSA)

2/ For 2004 and 2005, program figures reflect a change in classification of retirement allowances as part of exceptional spending. $3 /$ For 2005, interest payments include rescheduling agreements with commercial creditors under the enhanced HIPC Initiative. 4/ Exceptional expenditure includes spending for the Demobilization, Disarmament, and Reintegration (DDR) program, cost of the elections, payments for retirement allowances, repayments of domestic arrears, and payment for bank restructuring. 5/ For 2005, HIPC Initiative savings used for pro-poor wages and domestic investment were recorded under wages and domestic-financed investment.

$6 /$ Internal and external arrears.

7/ Underlying fiscal balance is defined as revenue minus expenditure and excluding interest on foreign debt, foreign-financed capital expenditures, all exceptional spending and repayment of domestic arrears. Difference between estimated figure for 2005 and the one reported in IMF Country Report No. 05/374 reflects the change in definition to exclude all exceptional spending. 
Table 3. Democratic Republic of the Congo: Summary of Central Government Operations, 2004-06 (Percent of GDP; unless indicated otherwise)

\begin{tabular}{|c|c|c|c|c|c|}
\hline & \multirow{2}{*}{$\begin{array}{r}2004 \\
\text { Est. }\end{array}$} & \multicolumn{2}{|c|}{2005} & \multicolumn{2}{|c|}{2006} \\
\hline & & $\begin{array}{l}\text { IMF Country } \\
\text { Report No. } \\
05 / 374\end{array}$ & Est. & $\begin{array}{l}\text { IMF Country } \\
\text { Report No. } \\
05 / 374\end{array}$ & Proj. \\
\hline Total revenue and grants & 11.5 & 19.9 & 16.8 & 18.9 & 22.0 \\
\hline Total revenue & 9.5 & 10.7 & 11.6 & 11.5 & 12.5 \\
\hline Customs and excise & 4.0 & 4.4 & 4.3 & 5.1 & 4.4 \\
\hline Direct and indirect taxes & 2.7 & 2.9 & 3.3 & 3.5 & 3.6 \\
\hline Petroleum (royalties and taxes) & 2.0 & 2.2 & 2.9 & 1.6 & 3.2 \\
\hline Other & 0.8 & 1.1 & 1.0 & 1.2 & 1.2 \\
\hline Total grants & 2.0 & 9.3 & 5.2 & 7.5 & 9.5 \\
\hline Budget grants & 0.1 & 0.9 & 0.2 & 0.0 & 1.0 \\
\hline Project grants & 1.0 & 5.6 & 2.2 & 3.6 & 5.3 \\
\hline HIPC Initiative assistance 1/ & 0.9 & 2.8 & 2.8 & 3.8 & 3.1 \\
\hline Total expenditure & 15.6 & 22.3 & 19.5 & 20.6 & 23.2 \\
\hline Current expenditure & 12.8 & 10.2 & 12.7 & 10.5 & 11.5 \\
\hline Wages 2/ & 3.6 & 3.7 & 4.4 & 4.0 & 4.7 \\
\hline Interest due 3/ & 3.6 & 3.2 & 3.4 & 2.9 & 3.2 \\
\hline Of which: on external debt & 3.3 & 3.1 & 2.9 & 2.9 & 2.9 \\
\hline Transfers and subsidies & 0.6 & 0.5 & 0.8 & 0.6 & 0.7 \\
\hline Other current expenditure & 5.0 & 2.9 & 4.2 & 3.0 & 2.9 \\
\hline Of which: centralized payments & 1.2 & 0.8 & 0.8 & 1.0 & 0.7 \\
\hline Capital expenditure & 2.8 & 6.9 & 3.4 & 8.4 & 6.2 \\
\hline Foreign-financed & 2.2 & 6.4 & 2.4 & 7.8 & 5.7 \\
\hline Domestic-financed & 0.6 & 0.5 & 1.0 & 0.6 & 0.5 \\
\hline Exceptional expenditure 4/ & 0.0 & 4.6 & 2.9 & 0.0 & 4.6 \\
\hline Foreign-financed & 0.0 & 4.1 & 1.9 & 0.0 & 3.4 \\
\hline Domestic-financed 5/ & 0.0 & 0.5 & 1.0 & 0.0 & 1.2 \\
\hline Other HIPC-related expenditure 6/ & 0.0 & 0.6 & 0.6 & 1.7 & 0.9 \\
\hline Overall fiscal balance (payment order basis) & -4.1 & -2.4 & -2.7 & -1.7 & -1.2 \\
\hline Change in arrears $5 /$ & -0.1 & 0.0 & -6.1 & 0.0 & -0.1 \\
\hline Float & 0.4 & -0.5 & 0.1 & 0.0 & 0.0 \\
\hline Central bank operational result & -0.7 & -0.4 & -0.4 & 0.0 & -0.3 \\
\hline Overall fiscal balance (cash basis, before interest rescheduling) & -4.4 & -3.3 & -9.1 & -2.2 & -1.5 \\
\hline Underlying fiscal balance $7 /$ & -0.9 & 1.6 & -0.2 & 1.0 & 2.2 \\
\hline Total financing & 3.4 & 3.3 & 9.1 & 2.2 & 1.5 \\
\hline Domestic financing & 0.0 & -0.8 & 7.8 & 0.0 & -0.8 \\
\hline Banking system & 0.0 & -0.8 & 1.7 & 0.0 & -0.4 \\
\hline Nonbanks & 0.0 & 0.0 & 0.8 & 0.0 & -0.4 \\
\hline Domestic debt relief & 0.0 & 0.0 & 5.3 & 0.0 & 0.0 \\
\hline Foreign financing & 3.4 & 4.1 & 1.5 & 0.4 & 1.5 \\
\hline Amortization due before debt relief & -3.3 & -4.4 & -4.5 & -5.8 & -5.6 \\
\hline Loan disbursement & 3.4 & 5.8 & 3.4 & 4.1 & 3.8 \\
\hline Budget loans & 2.3 & 1.6 & 1.5 & 0.0 & 0.0 \\
\hline Project-loans & 1.1 & 4.2 & 1.9 & 4.1 & 3.8 \\
\hline Debt relief before HIPC Initiative assistance & 3.3 & 2.6 & 2.5 & 2.1 & 3.2 \\
\hline Additional financing to be found & 0.0 & 0.0 & 0.0 & 1.7 & 0.8 \\
\hline \multicolumn{6}{|l|}{ Memorandum item: } \\
\hline GDP (in billions of CGF) & 2,601 & 3,380 & 3,366 & 3,814 & 3,868 \\
\hline
\end{tabular}

Sources: Congolese authorities and IMF staff estimates and projections.

1/ Reflects revised calculation of HIPC Initiative assistance from 2002-based Debt Sustainability Analysis (DSA).

2/ For 2004 and 2005, program figures reflect a change in classification of retirement allowances as part of exceptional spending. $3 /$ For 2005, interest payments include rescheduling agreements with commercial creditors under the enhanced HIPC Initiative.

4/ Exceptional expenditure includes spending for the Demobilization, Disarmament, and Reintegration (DDR) program, cost of the elections, payments for retirement allowances, repayments of domestic arrears, and payment for bank restructuring. $5 /$ Internal and external arrears.

6/ For 2005, HIPC Initiative savings used for pro-poor wages and domestic investment were recorded under wages and domestic-financed investment.

7/ Underlying fiscal balance is defined as revenue minus expenditure and excluding interest on foreign debt, foreign-financed capital expenditures, all exceptional spending and repayment of domestic arrears. Difference between estimated figure for 2005 and the one reported in IMF Country Report No. 05/374 reflects the change in definition to exclude all exceptional spending. 
Table 4. Democratic Republic of the Congo: Treasury Cash Flow 2006 (Millions of Congo francs)

\begin{tabular}{|c|c|c|c|c|}
\hline & $\begin{array}{r}\text { Q1 } \\
\text { Prel. }\end{array}$ & $\begin{array}{c}\text { Q2 } \\
\text { Proj. }\end{array}$ & $\begin{array}{l}\text { Q3 } \\
\text { Proj. }\end{array}$ & $\begin{array}{l}\text { Q4 } \\
\text { Proj. }\end{array}$ \\
\hline Total revenue and grants & 266,066 & 204,529 & 193,537 & 186,100 \\
\hline Total revenue & 129,442 & 118,879 & 122,314 & 112,139 \\
\hline Customs and excise & 42,645 & 40,757 & 45,687 & 40,662 \\
\hline Direct and indirect taxes & 36,922 & 32,678 & 36,954 & 34,444 \\
\hline Non tax revenue & 11,424 & 13,406 & 9,924 & 13,186 \\
\hline Petroleum & 38,451 & 32,038 & 29,749 & 23,846 \\
\hline Total grants & 136,624 & 85,650 & 71,222 & 73,961 \\
\hline Budget grants $5 /$ & 38,222 & 0 & 0 & 2,300 \\
\hline HIPC debt relief & 37,274 & 23,624 & 36,266 & 23,934 \\
\hline Total expenditure $1 /$ & 225,621 & 244,690 & 198,973 & 209,853 \\
\hline Current expenditure & 109,490 & 111,003 & 95,582 & 97,340 \\
\hline Wages & 44,604 & 46,105 & 44,302 & 45,138 \\
\hline Military and police & 9,284 & 8,416 & 8,271 & 7,113 \\
\hline Civilians & 35,320 & 37,689 & 36,031 & 38,025 \\
\hline Interest payments & 29,603 & 21,568 & 24,299 & 16,924 \\
\hline External debt (interest payment after debt relief) $2 /$ & 26,294 & 17,464 & 22,964 & 15,648 \\
\hline Domestic debt (interest payment) & 3,309 & 4,104 & 1,335 & 1,276 \\
\hline Other current expenditure & 27,552 & 33,257 & 20,767 & 31,079 \\
\hline Transfers and subsidies & 7,731 & 10,073 & 6,215 & 4,200 \\
\hline Capital expenditure & 22,496 & 71,933 & 68,174 & 78,048 \\
\hline Foreign financed & 21,040 & 60,033 & 64,252 & 75,716 \\
\hline Domestically financed & 1,457 & 11,900 & 3,922 & 2,332 \\
\hline Other HIPC-related expenditure & 955 & 5,464 & 3,415 & 24,316 \\
\hline Exceptional expenditures $3 /$ & 68,028 & 52,942 & 30,301 & 27,574 \\
\hline Float & 18,386 & 0 & 0 & $-18,386$ \\
\hline BCC treasury deficit & 5,539 & 2,000 & 1,500 & 961 \\
\hline Underlying fiscal balance (cash basis) 4/ & 19,183 & 4,628 & 40,859 & 21,223 \\
\hline Consolidated balance (cash basis) & 40,445 & $-40,160$ & $-5,436$ & $-23,754$ \\
\hline Total financing & $-40,445$ & 40,160 & 5,436 & 23,754 \\
\hline Domestic financing & $-17,989$ & 13,581 & 2,902 & $-27,535$ \\
\hline Foreign financing & $-22,456$ & 26,579 & 2,535 & 19,853 \\
\hline Amortization (net payment) 2/ & $-42,793$ & $-18,278$ & $-43,227$ & $-17,359$ \\
\hline Debt relief & $-14,698$ & $-8,109$ & $-14,309$ & $-8,446$ \\
\hline Amortization paid & $-28,095$ & $-10,169$ & $-28,918$ & $-8,912$ \\
\hline Loan financing & 13,621 & 45,047 & 49,677 & 39,822 \\
\hline Residual financing gap & 0 & 0 & 0 & 31,436 \\
\hline
\end{tabular}

Sources: Congolese authorities and staff estimates and projections.

$1 /$ Including domestic arrears and the balance of the BCC (for the latter,a minus sign corresponds to a surplus).

2/ Includes deposits into the HIPC account at the BCC.

3/ Exceptional expenditure include spending for the DDR program, cost of the elections, payments for retirement allowances, repayment of the domestic debt, and payment for bank restructuring.

4/ Underlying fiscal balance is defined as revenue minus expenditure and excluding interest on foreign debt, foreign-financed capital expenditures, all exceptional spending and repayment of domestic arrears. Difference between estimated figure for 2005 and the one reported in IMF Country Report No. 05/374 reflects the change in definition to exclude all exceptional spending

5/ In Q4, World Bank grant for education project classified as budget support. 
Table 5. Democratic Republic of the Congo: Monetary Survey, 2004-06

(At current exchange rates)

\begin{tabular}{|c|c|c|c|c|c|c|}
\hline & \multirow[t]{2}{*}{2004} & \multirow{2}{*}{$\begin{array}{c}2005 \\
\text { Dec } \\
\text { Prel. }\end{array}$} & \multicolumn{4}{|c|}{2006} \\
\hline & & & $\begin{array}{l}\text { Mar } \\
\text { Prel. }\end{array}$ & $\begin{array}{l}\text { Jun. } \\
\text { Proj. }\end{array}$ & $\begin{array}{l}\text { Sep. } \\
\text { Proj. }\end{array}$ & $\begin{array}{l}\text { Dec. } \\
\text { Proj. }\end{array}$ \\
\hline & \multicolumn{6}{|c|}{ (Billions of Congo francs) } \\
\hline Net foreign assets & -281.8 & -273.9 & -262.7 & -285.0 & -279.3 & -242.3 \\
\hline Net domestic assets & 504.0 & 551.0 & 564.1 & 605.8 & 601.6 & 579.0 \\
\hline Domestic credit & 68.9 & 148.0 & 134.9 & 160.6 & 161.3 & 143.6 \\
\hline Net credit to government & 27.2 & 84.6 & 66.6 & 81.7 & 84.6 & 69.1 \\
\hline Credit to the private sector & 39.9 & 63.0 & 67.3 & 76.7 & 74.7 & 72.8 \\
\hline Credit to parastatals & 1.8 & 0.4 & 1.0 & 2.2 & 2.0 & 1.7 \\
\hline Other items, net (including valuation change) & 435.1 & 403.0 & 429.2 & 445.2 & 440.3 & 435.4 \\
\hline Broad money (M2) & 215.3 & 267.3 & 288.9 & 310.3 & 310.2 & 322.9 \\
\hline Narrow money (M1) & 117.1 & 138.9 & 139.6 & 153.5 & 154.2 & 167.8 \\
\hline Currency in circulation & 101.5 & 119.9 & 120.0 & 123.6 & 125.8 & 140.9 \\
\hline Demand deposits & 15.6 & 19.0 & 19.7 & 29.9 & 28.4 & 26.9 \\
\hline Quasi money & 98.2 & 128.4 & 149.2 & 156.8 & 156.0 & 155.2 \\
\hline Time deposits in domestic currency & 0.4 & 0.4 & 0.5 & 0.7 & 1.2 & 1.8 \\
\hline Foreign currency deposits & 97.8 & 128.0 & 148.7 & 156.1 & 154.7 & 153.4 \\
\hline \multirow[t]{2}{*}{ Import deposits } & 7.0 & 9.8 & 12.5 & 10.5 & 12.1 & 13.7 \\
\hline & \multicolumn{6}{|c|}{ (Year-on-year change in percent) } \\
\hline Net foreign assets & -8.8 & 2.8 & 10.7 & -10.3 & -4.8 & 11.5 \\
\hline Net domestic assets & 29.5 & 9.3 & 0.1 & 18.6 & 14.2 & 5.1 \\
\hline Domestic credit & 40.0 & 115.0 & 42.0 & 56.6 & 56.8 & -3.0 \\
\hline Net credit to government & -8.0 & 210.8 & 47.8 & 56.3 & 71.8 & -18.3 \\
\hline Credit to the private sector & 105.5 & 58.1 & 36.9 & 53.9 & 40.8 & 15.5 \\
\hline Credit to parastatals & 688.3 & -75.3 & 34.0 & 370.8 & 275.8 & 283.1 \\
\hline Other items, net (including valuation change) & 28.0 & -7.4 & -8.4 & 9.0 & 3.9 & 8.1 \\
\hline Broad money (M2) & 72.9 & 24.2 & 11.6 & 27.4 & 25.0 & 20.8 \\
\hline Narrow money (M1) & 62.4 & 18.6 & 18.1 & 22.1 & 21.6 & 20.8 \\
\hline Currency in circulation & 60.7 & 18.2 & 17.2 & 12.7 & 12.9 & 17.5 \\
\hline Demand deposits & 74.2 & 21.4 & 23.6 & 86.0 & 84.6 & 41.8 \\
\hline Quasi money & 87.4 & 30.8 & 6.2 & 33.1 & 28.5 & 20.8 \\
\hline Time deposits in domestic currency & 12.8 & 5.3 & -39.6 & 99.3 & 177.3 & 361.7 \\
\hline Foreign currency deposits & 87.9 & 30.9 & 6.5 & 32.9 & 28.0 & 19.8 \\
\hline \multirow[t]{2}{*}{ Import deposits } & 23.9 & 41.2 & 18.9 & 19.5 & -0.3 & 39.4 \\
\hline & \multicolumn{6}{|c|}{$\begin{array}{l}\text { (Annual change in percent of } \\
\text { beginning-of-period broad money) }\end{array}$} \\
\hline Net foreign assets & -18.3 & 3.7 & 4.2 & -4.2 & -2.0 & 11.8 \\
\hline Net domestic assets & 92.3 & 21.8 & 4.9 & 20.5 & 18.9 & 10.5 \\
\hline Domestic credit & 15.8 & 36.8 & -4.9 & 4.7 & 4.9 & -1.7 \\
\hline Net credit to government & -1.9 & 26.6 & -6.7 & -1.1 & 0.0 & -5.8 \\
\hline Credit to the private sector & 16.4 & 10.8 & 1.6 & 5.1 & 4.4 & 3.7 \\
\hline Credit to parastatals & 1.3 & -0.6 & 0.2 & 0.7 & 0.6 & 0.5 \\
\hline Other items, net (including valuation change) & 76.5 & -14.9 & 9.8 & 15.8 & 14.0 & 12.1 \\
\hline Broad money (M2) & 72.9 & 24.2 & 8.1 & 16.1 & 16.1 & 20.8 \\
\hline Narrow money (M1) & 36.1 & 10.1 & 0.3 & 5.5 & 5.7 & 10.8 \\
\hline Currency in circulation & 30.8 & 8.6 & 0.0 & 1.4 & 2.2 & 7.8 \\
\hline Demand deposits & 5.3 & 1.6 & 0.3 & 4.1 & 3.5 & 3.0 \\
\hline Quasi money & 36.8 & 14.0 & 7.8 & 10.6 & 10.3 & 10.0 \\
\hline Time deposits in domestic currency & 0.0 & 0.0 & 0.1 & 0.1 & 0.3 & 0.5 \\
\hline Foreign currency deposits & 36.8 & 14.0 & 7.7 & 10.5 & 10.0 & 9.5 \\
\hline Import deposits & 1.1 & 1.3 & 1.0 & 0.3 & 0.9 & 1.4 \\
\hline \multicolumn{7}{|l|}{ Memorandum items: } \\
\hline Nominal GDP (billions of Congo francs) & 2,601 & 3,366 & 3,868 & $\ldots$ & $\ldots$ & 3,868 \\
\hline Velocity (GDP/broad money) & 12.1 & 12.6 & 13.4 & $\ldots$ & $\ldots$ & 12.0 \\
\hline Foreign currency deposits (percent of M2) & 45.4 & 47.9 & 51.5 & 50.3 & 49.9 & 47.5 \\
\hline Foreign currency deposits (percent of total deposits) & 86.0 & 86.9 & 88.0 & 83.6 & 83.9 & 84.3 \\
\hline
\end{tabular}

Sources: Congolese authorities and IMF staff estimates and projections. 
Table 6. Democratic Republic of the Congo: Accounts of the Central Bank of the Congo, 2004-06 (At current exchange rates)

\begin{tabular}{|c|c|c|c|c|c|c|}
\hline & \multirow[t]{2}{*}{2004} & \multirow{2}{*}{$\begin{array}{c}2005 \\
\text { Dec } \\
\text { Prel. }\end{array}$} & \multicolumn{4}{|c|}{2006} \\
\hline & & & $\begin{array}{l}\text { Mar } \\
\text { Prel. }\end{array}$ & $\begin{array}{l}\text { Jun. } \\
\text { Proj. }\end{array}$ & $\begin{array}{l}\text { Sep. } \\
\text { Proj. }\end{array}$ & $\begin{array}{l}\text { Dec. } \\
\text { Proj. }\end{array}$ \\
\hline & \multicolumn{6}{|c|}{ (Billions of Congo francs) } \\
\hline Net foreign assets & -347.6 & -357.8 & -347.0 & -382.1 & -378.6 & -343.8 \\
\hline Gross offical reserves (in millions of US dollars) & 236.2 & 131.2 & 174.8 & 150.4 & 163.5 & 246.9 \\
\hline Foreign assets & 104.9 & 56.6 & 76.6 & 68.4 & 77.8 & 118.5 \\
\hline Foreign liabilities & 452.5 & 414.4 & 423.6 & 450.5 & 456.4 & 462.2 \\
\hline Net domestic assets & 470.3 & 496.0 & 492.3 & 530.6 & 526.9 & 504.9 \\
\hline Domestic credit & 52.2 & 123.2 & 107.0 & 120.0 & 117.8 & 97.2 \\
\hline Net credit to government & 38.4 & 103.3 & 90.3 & 103.2 & 104.7 & 87.8 \\
\hline Credit to the private sector & 1.2 & 0.0 & 0.0 & 0.2 & 0.1 & 0.0 \\
\hline Credit to parastatals & 0.0 & 0.0 & 0.0 & 0.0 & 0.0 & 0.0 \\
\hline Claims on deposit money banks & 12.6 & 20.0 & 16.7 & 16.6 & 13.0 & 9.4 \\
\hline Other items, net & 418.1 & 372.7 & 385.3 & 410.6 & 409.2 & 407.8 \\
\hline Base money & 122.7 & 138.2 & 145.3 & 148.5 & 148.4 & 161.1 \\
\hline Narrow base money & 111.9 & 129.2 & 135.0 & 140.6 & 138.9 & 150.0 \\
\hline Currency in circulation & 105.9 & 123.8 & 125.6 & 129.4 & 130.1 & 143.7 \\
\hline Deposits at the central bank & 5.8 & 5.3 & 9.1 & 10.9 & 8.5 & 6.1 \\
\hline Private sector deposits & 0.1 & 0.1 & 0.1 & 0.1 & 0.1 & 0.1 \\
\hline Parastatal deposits & 0.2 & 0.1 & 0.1 & 0.1 & 0.1 & 0.1 \\
\hline Foreign currency deposits & 6.1 & 5.6 & 6.8 & 5.0 & 6.0 & 7.0 \\
\hline \multirow[t]{2}{*}{ Import deposits } & 4.7 & 3.4 & 3.5 & 2.9 & 3.5 & 4.2 \\
\hline & \multicolumn{6}{|c|}{ (Year-on-year change in percent) } \\
\hline Net foreign assets & -17.9 & -2.9 & 14.0 & -15.6 & -9.3 & 3.9 \\
\hline Net domestic assets & 27.1 & 5.5 & -6.3 & 15.1 & 11.1 & 1.8 \\
\hline Domestic credit & 22.8 & 136.3 & 54.2 & 46.4 & 62.9 & -21.2 \\
\hline Net credit to government & 4.0 & 169.1 & 55.0 & 52.5 & 66.9 & -15.0 \\
\hline Credit to the private sector & -30.3 & -99.6 & -96.0 & -75.3 & -87.5 & 0.0 \\
\hline Credit to parastatals & 0.0 & 4.1 & 12.3 & 156.8 & 78.4 & 0.0 \\
\hline Claims on deposit money banks & 223.1 & 58.4 & 64.5 & 22.0 & 46.3 & -53.1 \\
\hline Other items, net & 27.6 & -10.8 & -15.5 & 8.4 & 1.8 & 9.4 \\
\hline Base money & 63.2 & 12.7 & 19.3 & 14.0 & 15.9 & 16.6 \\
\hline Narrow base money & 65.3 & 15.5 & 21.8 & 16.1 & 15.9 & 16.1 \\
\hline Currency in circulation & 60.7 & 16.9 & 18.4 & 14.8 & 13.2 & 16.1 \\
\hline Deposits at the central bank & 292.2 & -9.1 & 115.3 & 35.5 & 99.3 & 15.2 \\
\hline Private sector deposits & -80.2 & 46.6 & -52.5 & -20.8 & -63.7 & 0.0 \\
\hline Parastatal deposits & 125.7 & -53.8 & -34.6 & -14.9 & -54.3 & 0.0 \\
\hline Foreign currency deposits & 53.2 & -7.4 & 31.7 & 10.4 & 59.4 & 24.2 \\
\hline \multirow[t]{2}{*}{ Import deposits } & 35.5 & -28.3 & -40.3 & -37.3 & -21.6 & 24.6 \\
\hline & \multicolumn{6}{|c|}{$\begin{array}{l}\text { (Annual change in percent of } \\
\text { beginning-of-period base money) }\end{array}$} \\
\hline Net foreign assets & -70.1 & -8.3 & 7.8 & -17.6 & -15.0 & 10.1 \\
\hline Net domestic assets & 133.4 & 21.0 & -2.7 & 25.0 & 22.4 & 6.5 \\
\hline Domestic credit & 12.9 & 57.9 & -11.8 & -2.4 & -4.0 & -18.9 \\
\hline Net credit to government & 2.0 & 52.9 & -9.4 & -0.1 & 1.0 & -11.2 \\
\hline Credit to the private sector & -0.7 & -1.0 & 0.0 & 0.1 & 0.1 & 0.0 \\
\hline Credit to parastatals & 0.0 & 0.0 & 0.0 & 0.0 & 0.0 & 0.0 \\
\hline Claims on deposit money banks & 11.6 & 6.0 & -2.4 & -2.4 & -5.1 & -7.7 \\
\hline Other items, net & 120.5 & -37.0 & 9.1 & 27.4 & 26.4 & 25.3 \\
\hline Base money & 63.2 & 12.7 & 5.1 & 7.4 & 7.4 & 16.6 \\
\hline Narrow base money & 58.8 & 14.1 & 4.2 & 8.2 & 7.0 & 15.0 \\
\hline Currency in circulation & 53.2 & 14.6 & 1.3 & 4.0 & 4.6 & 14.4 \\
\hline Deposits at the central bank & 5.7 & -0.4 & 2.8 & 4.1 & 2.3 & 0.6 \\
\hline Private sector deposits & -0.3 & 0.0 & 0.0 & 0.0 & 0.0 & 0.0 \\
\hline Parastatal deposits & 0.1 & -0.1 & 0.0 & 0.0 & 0.0 & 0.0 \\
\hline Foreign currency deposits & 2.8 & -0.4 & 0.8 & -0.4 & 0.3 & 1.0 \\
\hline Import deposits & 1.6 & -1.1 & 0.1 & -0.3 & 0.1 & 0.6 \\
\hline
\end{tabular}

Sources: Congolese authorities and IMF staff estimates and projections. 
Table 7. Democratic Republic of the Congo: Balance of Payments Summary, 2004-09

\begin{tabular}{|c|c|c|c|c|c|c|c|c|}
\hline & 2004 & 2005 & & 2006 & & 2007 & 2008 & 2009 \\
\hline & Est. & $\begin{array}{c}\text { IMF Country } \\
\text { Report No. } \\
05 / 374\end{array}$ & Proj. & $\begin{array}{c}\text { IMF Country } \\
\text { Report No. } \\
05 / 374\end{array}$ & Proj. & Proj. & Proj. & Proj. \\
\hline & & & ns of U.S & dollars; unless & erwise in & ted) & & \\
\hline Current account & -375 & -354 & -345 & -618 & -404 & -393 & -370 & -473 \\
\hline Merchandise trade & -244 & -423 & -198 & -562 & -468 & -342 & -436 & -473 \\
\hline Exports, f.o.b. & 1,813 & 2,042 & 2,050 & 2,044 & 2,366 & 2,650 & 2,739 & 2,866 \\
\hline Of which: diamonds & 828 & 989 & 955 & 1,154 & 1,087 & 1,245 & 1,362 & 1,462 \\
\hline Of which: oil exports & 360 & 409 & 453 & 366 & 494 & 519 & 517 & 511 \\
\hline Imports, f.o.b. & $-2,056$ & $-2,465$ & $-2,248$ & $-2,607$ & $-2,834$ & $-2,992$ & $-3,175$ & $-3,339$ \\
\hline Of which: aid-related imports & -306 & -565 & -309 & -499 & -649 & -527 & -521 & -484 \\
\hline Services & -333 & -499 & -351 & -466 & -533 & -579 & -603 & -603 \\
\hline Receipts & 172 & 191 & 190 & 196 & 195 & 196 & 212 & 233 \\
\hline Expenditure & -505 & -690 & -541 & -662 & -728 & -775 & -815 & -836 \\
\hline Of which: aid-related imports & -112 & -214 & -118 & -184 & -240 & -186 & -190 & -147 \\
\hline Income & -294 & -337 & -344 & -356 & -400 & -356 & -251 & -214 \\
\hline Receipts & 91 & 105 & 105 & 123 & 124 & 150 & 192 & 261 \\
\hline Expenditure & -385 & -442 & -449 & -479 & -524 & -506 & -443 & -474 \\
\hline Of which: interest payments $1 /$ & -221 & -220 & -238 & -231 & -251 & -233 & -227 & -215 \\
\hline Current transfers & 496 & 905 & 548 & 766 & 997 & 883 & 921 & 817 \\
\hline Of which: official aid & 432 & 768 & 412 & 583 & 838 & 693 & 705 & 606 \\
\hline Capital and financial account & -124 & 132 & -12 & 106 & 5 & -257 & -167 & 61 \\
\hline Official capital & -11 & 97 & -30 & -126 & -149 & -170 & -186 & -181 \\
\hline Gross disbursements & 220 & 402 & 253 & 322 & 322 & 330 & 301 & 329 \\
\hline Of which: net new financing & 220 & 402 & 253 & 322 & 322 & 330 & 301 & 329 \\
\hline Scheduled amortization 2/ & -230 & -305 & -283 & -449 & -471 & -500 & -488 & -510 \\
\hline Private capital (net) $3 /$ & -113 & 34 & 18 & 232 & 154 & -86 & 19 & 241 \\
\hline Of which: foreign direct investment & 435 & 551 & 405 & 633 & 496 & 675 & 741 & 814 \\
\hline Balance before errors and omissions & -499 & -223 & -357 & -512 & -399 & -650 & -537 & -412 \\
\hline Errors and omissions & 242 & 0 & 0 & 0 & 0 & 0 & 0 & 0 \\
\hline Overall balance & -257 & -223 & -357 & -512 & -399 & -650 & -537 & -412 \\
\hline Financing & -393 & -865 & -99 & -83 & -432 & -119 & -252 & -390 \\
\hline Net change in non-Fund arrears & -332 & -707 & -99 & 0 & -302 & 0 & 0 & 0 \\
\hline Net banking sector reserves (increase, - ) & -61 & -157 & 1 & -83 & -130 & -119 & -252 & -390 \\
\hline Of which: net Fund credit & 69 & 37 & 37 & 39 & 0 & -62 & -124 & -140 \\
\hline Financing need before exceptional assistance & -649 & $-1,087$ & -455 & -596 & -831 & -769 & -789 & -802 \\
\hline Exceptional financing & 649 & 1,087 & 467 & 461 & 762 & 564 & 620 & 621 \\
\hline Consolidation of arrears & 344 & 707 & 100 & 0 & 302 & 0 & 0 & 0 \\
\hline Debt relief on current debt service & 305 & 380 & 367 & 461 & 461 & 564 & 620 & 621 \\
\hline Residual financing need (overfinancing, + ) & 0 & 0 & 12 & -134 & -68 & -205 & -169 & -181 \\
\hline & & (In per & nt of $G D$ & unless otherwis & ndicated) & & & \\
\hline Memorandum items: & & & & & & & & \\
\hline Debt service, after debt relief (percentage of exports of goods and services) $4 /$ & 8.2 & 6.5 & 6.9 & 9.7 & 9.8 & 11.8 & 9.8 & 10.2 \\
\hline Current account balance, incl. grants, before debt relief & -5.7 & -5.1 & -4.9 & -7.9 & -4.8 & -4.3 & -3.7 & -4.4 \\
\hline Current account balance, excl. official transfers, before debt relief & -12.3 & -16.2 & -10.7 & -15.4 & -14.8 & -11.9 & -10.9 & -10.1 \\
\hline Current account balance, incl. grants, after debt relief & -4.3 & -2.2 & -1.9 & -4.1 & -0.9 & -0.1 & -0.1 & -0.9 \\
\hline Current account balance, excl. grants, after debt relief & -10.9 & -13.4 & -7.7 & -11.6 & -10.9 & -7.7 & -7.2 & -6.6 \\
\hline Gross official reserves (in millions of U.S. dollars) & 236.2 & 360.2 & 131.2 & 470.2 & 246.8 & 300.3 & 338.2 & 424.5 \\
\hline Weeks of non-aid-related imports of goods and services & 5.2 & 7.2 & 2.6 & 8.5 & 4.2 & 4.8 & 5.0 & 5.9 \\
\hline
\end{tabular}

Sources: Congolese authorities and IMF staff estimates and projections.

1/ Including interest due to the IMF.

2/ Excluding repayments to the IMF.

3/ Including unrecorded transactions. The latter may be substantial given the weak state of statistics.

4/ It takes into account the Cologne flow rescheduling and the capitalization of moratorium interest, the additional debt service relief beyond the Cologne flow rescheduling and the grants provided by multilateral creditors as interim HIPC assistance and assumes a completion point in 2007. 


\section{APPENDIX I \\ TRANSLATED FROM FRENCH}

Kinshasa, June 29, 2006

Mr. Rodrigo de Rato

Managing Director

International Monetary Fund

Washington, D.C., 20431

Dear Mr. de Rato:

1. The Democratic Republic of the Congo has implemented an economic program that has been supported since 2002 by the International Monetary Fund under the Poverty Reduction and Growth Facility (PRGF). This program has enabled us to begin to rebuild our economy, restore growth, start reducing poverty, and curb inflation.

2. The government acknowledges the difficulties that have prevented the completion of the last program review before the PRGF arrangement expired on March 31, 2006. These difficulties explain the nonobservance of the performance criterion for net credit to the public sector at the end of September and three key quantitative indicators at the end of December 2005. Slow implementation of structural reforms also led to the nonobservance of four September structural performance criteria.

3. Nevertheless, the government remains determined to step up its efforts to preserve macroeconomic stability and to continue rebuilding the country, particularly during the final phase of the political transition. For the first time in its history, the Congolese people are preparing to select their future leaders in free, democratic and transparent elections organized with the help of the international community.

4. For these reasons, as dialogue continues between the government and the International Monetary Fund, we are requesting your assistance in implementing a staffmonitored program for the period April 1-December 31, 2006.

5. The success of the staff-monitored program should lay the foundation for a new arrangement with the International Monetary Fund under the PRGF that will enable the Democratic Republic of the Congo to continue benefiting from debt relief under the enhanced HIPC Initiative and the Multilateral Debt Relief Initiative. 
6. The government is committed to implementing the policies and measures set out in the attached Memorandum so as to achieve the objectives of the economic and financial program for 2006. It will submit all information requested by the International Monetary Fund on program implementation. In addition, the government is prepared to take any additional measures that may prove necessary to that end, after consulting with Fund staff. As in the past, the government authorizes the publication of this letter and the attached Memorandum of Economic and Financial Policies.

Sincerely yours,

/s/

Joseph Kabila

President of the Democratic Republic of the Congo 


\title{
APPENDIX I \\ ATTACHMENT I \\ TRANSLATED FROM FRENCH
}

\author{
DEMOCRATIC REPUblic OF THE CONGO \\ Memorandum on Economic AND Financial Policies For APRIL-DeCEMber 2006
}

Kinshasa, June 29, 2006

\section{INTRODUCTION}

1. This memorandum describes the government's economic program (Programme Relais de Consolidation (PRC)) for the period April-December 2006 that the authorities will monitor with the assistance of Fund staff. The program aims at establishing a strong record of macroeconomic and structural policy implementation following the easing of policies at the end of last year and the expiration of the Poverty Reduction and Growth Facility (PRGF) arrangement in March 2006. Its successful implementation will set the stage for discussions on a program to be supported by a successor PRGF arrangement after the forthcoming elections and the formation of a new government. The DRC government is very keen on implementing a PRGF-supported program with a view to be granted debt relief under the enhanced HIPC Initiative and the Multilateral Debt Relief Initiative (MDRI) in the second half of 2007.

2. The government is firmly committed to a stable macroeconomic environment during the first free, democratic, and transparent elections to be held in the DRC, which will mark the end of the political transition. The parliamentary and the first round of the presidential elections are to be held on July 30, 2006. If necessary, a second round of the presidential election together with local elections will take place thereafter and be followed by the formation of a new government. The government is confident that security will be maintained in the coming months with the assistance of troops from the United Nations (MONUC) and the European Union (EUFOR).

\section{RECENT ECONOMIC DEVELOPMENTS}

3. Macroeconomic developments were mixed in 2005. Real GDP growth held steady at 6.5 percent in 2005, mainly on account of strong performance in mining, construction, telecommunications, and wholesale trade. After peaking at 27 percent in August, 12-month inflation receded gradually to 21.3 percent at the end of 2005 . The decline was facilitated by an appreciation of the Congo franc vis-à-vis the US dollar since last August, which resulted from an increased use of foreign exchange reserves to contain money expansion in the face of increasing government spending. Hence, international reserves dropped to 2.6 weeks of imports by end-2005, well below the target of 7 weeks under the program. 
4. Difficulties in controlling government spending led to a deteriorating fiscal situation in spite of a strong revenue performance. The underlying fiscal balance registered a deficit equivalent to 0.2 percent of GDP, whereas a surplus of 1.6 percent of GDP had been targeted. ${ }^{2}$ The overrun in current spending amounted to 2.5 percent of GDP and was mainly due to increases in wage benefits granted in October and November to ease rising social tensions and to difficulties in containing sovereignty outlays (political institutions and the military). The overall fiscal deficit, however, was only slightly larger than anticipated because of low foreign-financed capital expenditure and outlays related to the elections, which were postponed to 2006.

5. Monetary aggregates grew broadly as anticipated in 2005. Monetary expansion was contained by using foreign exchange reserves to offset a large increase in net bank credit to the government. In particular, the share of government spending directly paid in foreign currency rose significantly during the last five months of the year. The sharp increase in the interest rates of the $\mathrm{BCC}$ also contributed to a rise in the outstanding amount of treasury bills issued by the BCC to CGF4.9 billion in 2005 (0.1 percent of GDP).

6. The macroeconomic situation remained fragile in the first four months of 2006. Improved government revenue collection, especially receipts from oil production, led to a strengthening of the fiscal position and an increase in international reserves in March 2006. This was accompanied by a further decline in inflation and a stable exchange rate. However, in April, rising outlays for political institutions and the military financed through bank credit (CF15 billion or 0.4 percent of GDP) resulted in a rapid increase in broad money, which the $\mathrm{BCC}$ had difficulties containing. This in turn led to a depreciation of the Congo franc and an increase in inflation, which averaged 13 percent annualized in the first five months of the year.

\section{MACROECONOMIC FRAMEWORK AND POLICIES FOR 2006}

7. The government is determined to preserve the progress made over the past three years in improving the macroeconomic situation and reforming the economy. It is

keenly aware of the need to maintain tight policies during the electoral period to avoid a flare up in inflation and a depreciating currency. The authorities also intend to continue implementing structural reforms essential to meeting macroeconomic objectives. These include (i) improving public resource management and enhancing the quality and composition of spending; (ii) strengthening the management of the payroll and the civil service; (iii) enhancing the transparency of government operations, and (iv) continuing to

\footnotetext{
${ }^{2}$ The underlying fiscal balance excludes grants, all foreign financed expenditures, external interest payments, all outlays related to the political transition, and settlements of domestic arrears.
} 
implement measures to restructure and strengthen central bank operations. In addition, the government intends to adopt the PRSP before the end of June 2006.

8. The macroeconomic objectives for 2006 are broadly in line with the mediumterm framework underpinning the last PRGF arrangement. Real GDP is to grow by 6.5 percent as economic activity recovers steadily, and 12-month inflation is targeted to less than 10 percent. The target for international reserves has been set at close to 4 weeks of projected imports of nonaid-related goods and services by yearend. However, recognizing that the reserve level is low, the BCC will make every effort to increase reserves further while keeping base money expansion in line with projected nominal GDP growth. Meanwhile, the government will tighten fiscal policy while maintaining social spending so as not to have recourse to domestic bank financing.

9. Fiscal policy aims at improving the underlying fiscal balance by almost 2 percentage points of GDP in 2006. This is to be achieved by containing expenditure and strengthening tax and nontax administration to raise government revenue. Government revenue is projected to increase to above 12 percent of GDP on current trends. Conversely, government underlying expenditure - excluding foreign interest payments, foreign financed capital expenditure, and outlays related to the political transition-is projected to decline to 12.8 percent of GDP in 2006 from 14.1 percent in 2005. As a result, the budget deficit will narrow to 1.5 percent of GDP in 2006 from 2.7 percent in 2005.

10. The government does not intend to have recourse to domestic bank financing to cover the fiscal deficit in $\mathbf{2 0 0 6}$. The budget is fully financed until September 2006, but there remains a financing gap equivalent to 0.8 percent of GDP (or US\$68 million) in the last quarter. So the government is pursuing discussions with development partners on budget support and on ways to avoid difficulties in serving its external debt. At the time of the first review under the program, the government will review the situation with Fund staff and if necessary will discuss additional measures to ensure that the budget is financed without recourse to domestic bank credit. Were the government to secure external budget support higher than is required to cover the financing gap, up to 50 percent of the excess would be used to increase poverty-reducing outlays.

\section{To raise government revenue, the authorities will continue to improve} transparency and combat fraud in tax administration. For the customs office, OFIDA, measures to be taken include (i) implementing the presidential decree and the manual of procedures making OFIDA the sole agency collecting taxes and duties in the port of Matadi before the end of June 2006; and (ii) strengthening pre-shipment inspection and preparing a monthly report reconciling data from OFIDA with those of the firm in charge of preshipment inspection. The government will also seek financing to render operational the customs office and the one-stop window at Kasumbalesa in the Katanga province.

12. For the agency in charge of internal taxation (DGI), the measures include (i) adoption and implementation by June 2006 of the ministerial decree requiring the use of a single 
taxpayer identification number for all commercial and financial operations and for all tax payments; and (ii) opening new tax centers for small- and medium-scale enterprises in Lumumbashi by August 2006 and in Goma by December 2006. For the department in charge of nontax revenue (DGRAD), the main measures are (i) application of all tax provisions of the forestry and mining codes; (ii) adoption and implementation of the five remaining interministerial decrees setting the duties and fees to be collected by the DGRAD on behalf of ministries of interior, foreign affairs, transport and communications, energy/petroleum products, and environment; (iii) reduction of the shares of revenue earmarked for autonomous agencies and their replacement by budgeted transfer payments; and (iv) implementation of the laws concerning payments of dividends by public enterprises.

13. The authorities will also take the following measures:

- $\quad$ Raise revenue collected from the excise tax on petroleum products to its 2005 level by progressively increasing the taxable base to the import price from April to December 2006 and adjusting retail prices accordingly;

- Impose a moratorium on new joint ventures involving public enterprises, and transfer payments of signing bonuses expected in 2006 to the treasury;

- Grant no new tax exemptions until the end of 2006 and recognize only exemptions already granted under the investment, mining, and forestry codes;

- $\quad$ Request the DGI and the DGRAD to audit the 2005 accounts of the petroleum companies and submit a preliminary report by the end of September 2006. The government has also requested World Bank assistance for a legal audit of its contractual agreements with the petroleum companies and for a study that would improve the monitoring and control of petroleum production in DRC.

14. The government will cap the wage bill at 4.7 percent of GDP (CGF180 billion) in 2006. No increases in salary or benefits will be granted this year beyond what was done last fall. In addition, to improve civil service management, the government will complete a review and an updating of civil service administrative files by the end of September and revise the payroll accordingly. A new simplified payroll system (PTS) is also to become operational in September 2006. Control over the military payroll will be strengthened with the assistance of development partners. 
15. With respect to nonwage expenditure, the government will strictly adhere to budget allocations and comply with the quarterly spending plan for each ministry. The plan is integrated into the computerized expenditure procedures to prevent overspending; monthly revisions will ensure the plan's consistency with expected government resources and monetary programming objectives. The authorities will reduce the use of budget advances and will grant new advances only once the use of previous ones has been documented.

16. To improve the transparency of budget execution, the government will (i) post on the Internet a summary of the monthly cash flow and spending plans for the following three months and the actual monthly outcomes for each ministry starting in May 2006; (ii) starting in June 2006, submit to the Council of Ministers at the end of each month a report on outlays for travel and gasoline incurred by each ministry, as well as on budget advances; (iii) produce the treasury accounts for December 2005 on the new simplified double-entry accounting system implemented at the Ministry of Finance in June 2006; and (iv) complete in September 2006 a financial audit of expenditure financed through HIPC savings deposited in an account at the BCC during 2003-05.

17. The objective of monetary policy will remain to contain inflation while building up international reserves. Taking into account the volatility of the demand for money, the weakness of monetary policy instruments, and the dollarization of the economy, the authorities have assumed for monetary programming purposes a prudent increase in base money of 16.6 percent and in broad money of 20.8 percent in 2006 . These assumptions will be reassessed at the time of the first review. The BCC has already increased its base rate by 3 percentage points to mop up increased commercial banks' free reserves at the BCC and reduce pressures on the exchange rate in May. To strengthen the conduct of monetary policy, and more generally of its operations, the BCC will continue implementing the recommendations of Fund technical assistance summarized in Table 3. Before September 2006, an international audit firm will be selected through a bidding process to conduct a study of central bank organization before end-December 2006. The conclusion of the audit will be used to prepare a plan to restructure and recapitalize the BCC.

18. The BCC will seek to limit its operating deficit to CGF10 billion in 2006. To that end, the BCC prepared in May 2006 a monthly budget for each department and will submit a monthly report on its execution to its Board of Directors within 30 days of each month-end.

\section{The BCC will ensure that the takeover of l'Union des Banques Congolaises} (UBC) by the Banque Congolaise (BC) maintains the integrity of the banking system and the government will assess the cost to the budget. To this end, an international audit firm was hired to (i) assess UBC's losses as of June 15, 2006, currently estimated at US\$13 million; and (ii) confirm the amount of the severance package paid to UBC personnel-estimated at US\$2.7 million. In addition, the accounts of the $\mathrm{BC}$ will be audited taking into consideration the takeover of the UBC. In case the UBC's losses exceed

US\$13 million, the government will discuss with the $\mathrm{BC}$ how the additional amount will be 
financed so as to avoid further budgetary implications. On the basis of these audits, the government will prepare a note explaining the direct and indirect costs of the operation and its implications for fiscal and monetary policies, which will be discussed with Fund and Bank staffs. The government will also set September 30, 2006 as a deadline to report claims against UBC and discuss with Bank and Fund staffs how to deal with them. In addition, the Central Bank will maintain its three representatives currently with UBC management to closely monitor the implementation of the BC's business plan until the situation of the $\mathrm{BC}$ is stabilized.

\section{PRogram MONITORING}

20. The Interministerial Committee Responsible for Monitoring Programs Concluded with International Financial Institutions (CISPI), chaired by the Minister of Finance, and the Interministerial Commission Responsible for Formulation of the Poverty Reduction Strategy, chaired by the Minister of Planning, will continue to implement the measures of the Government Economic Program and the poverty reduction strategy. The work of the two commissions will be supported by the Economic and Financial Sub-Committee comprising the Minister of Finance, the Minister of Budget and the Governor of the BCC, assisted by a technical committee (the technical troika). To monitor program implementation, quarterly quantitative and structural indicators have been set and are reflected in the attached tables. The government will review program implementation with the Fund staff on a quarterly basis. The government's Letter of Intent and the Memorandum on Economic and Financial Policies will be broadly disseminated.

21. The monitoring of progress with implementing the program outlined above will be based on the quantitative and structural benchmarks provided in Tables 2 and 5 . End-June and end-September 2006 are test dates for the quantitative targets. 
Table 1. Democratic Republic of the Congo: Performance Criteria and Structural Benchmarks, 2005

Status

\section{Structural performance criteria}

Presidential decree specifying that OFIDA (Customs and Excise Office) is the sole agency operating the one-stop windows and valuing goods at customs facilities.

With respect to government accounting, implementation of a simplified double-entry accounting system by the treasury and government accounting departments of the Ministry of Finance, including their redeployment, review of procedures, and staff training.

Adoption by the government of a strategy to restructure the BCC balance sheet.

Application by the Ministry of Defense and the Ministry of Budget of the findings of the survey of military personnel in Kinshasa to the August 2005 payroll.

\section{Structural benchmarks}

Inventory and quantification of taxes and duties collected in favor of parastatals (such as ONATRA, OCC, OGEFREM, and CEEC).

Implementation of the simplified temporary payroll procedure, restoration of budgetary and administrative supervision, ensuring that paymasters (comptables payeurs) report to the Treasury.
End-September 2005

Implemented December 30, 2005

End-September 2005

Implemented June 2006

End-September 2005

End-September 2005

End-September 2005

End-September 2005
Partially implemented; requires an organizational audit of the BCC to be completed at end 2006

Superseded by assistance provided by the European Union to strengthen controls over military budget.

Done

To be implemented by September 2006 


\title{
Table 2. Democratic Republic of the Congo: Prior Actions and Structural Benchmarks, 2006
}

\author{
Measures
}

Timetable

\section{A. Prior actions}

1. Finalize monthly cash flow and spending plans for June-September 2006 and post them on the websites of the Ministry of Finance and the Ministry of Budget.

2. Post on websites the execution of cash flow and spending plans for March and April 2006.

3. Adopt manual of customs procedures by interministerial decree (ministries of finances, external trade, and transportation).

4. Sign ministerial decree endorsing contract between preshipment inspection firm and OFIDA.

5. Sign interministerial decree rendering compulsory the use of the new single taxpayer identification number for commercial, financial, and fiscal operations.

6. Sign the five interministerial decrees fixing the rate of fees to be collected by the nontax administration (DGRAD) for the ministries in charge of interior/police, foreign affairs, transport and communications, petroleum production, and environment.

7. Cap the share of earmarked funds to be transferred to autonomous agencies to agreed amounts.

\section{B. Structural benchmarks}

8. Audits by a internationally known auditing firm of the accounts of Union des Banques Congolaises (UBC) as of June 15, 2006 and of the accounts of Banque Congolaise that would result from the takeover of UBC. The audits will determine the total fiscal cost of the merger and will be the basis for a note to be jointly prepared by the BCC and the Ministry of Finance to explain how the government will finance this operation.

9. Implement new simplified payroll system taking into account results from census.

End-August 2006

10. Complete audit of use of HIPC funds.

End-September 2006

11. Select international audit firm to conduct an organizational audit of the BCC. 
Table 3. Democratic Republic of the Congo: Summary of Action Plan for the Central Bank of the Congo (BCC), 2006

Measures

1. Complete the reorganization of the Treasury Department of the BCC based on the recommendations of the National Bank of Belgium and the IMF.

2. Prepare a monthly table (Table 6 ) to track bank liquidity that is consistent with the BCC balance sheet data, including data from the provincial agencies (current and projections).

3. Keep foreign exchange accounts of the BCC in four domestic banks with total balances limited to US $\$ 5.0$ millions.

4. Implement software for the foreign exchange trading room, adopt and implement trading room procedures manual, and establish the related Operations Committee and Risk Committee.

5. Prepare BCC's accounts using new accounting software.

6. Produce a monthly cash-flow plan for the BCC, including a spending plan for each department, consistent with the objective of limiting the operating deficit to CF 10 billion, and submit a monthly report on its execution to the board of directors of the BCC.

7. Create a centralized database of monetary and financial indicators needed to conduct monetary policy.

8. Prepare a plan for restructuring or liquidating the savings bank CADECO and the deposit money bank UBC, with a view to reducing risk to the banking system.

9. Create and disseminate a Code of Ethics and Conduct for inspectors in the Financial Intermediaries Supervision Directorate (DSIF).
Status

September 2006

June 2006

June 2006

June 2006

December 2006

June 2006

June 2006

June 2006

June 2006 


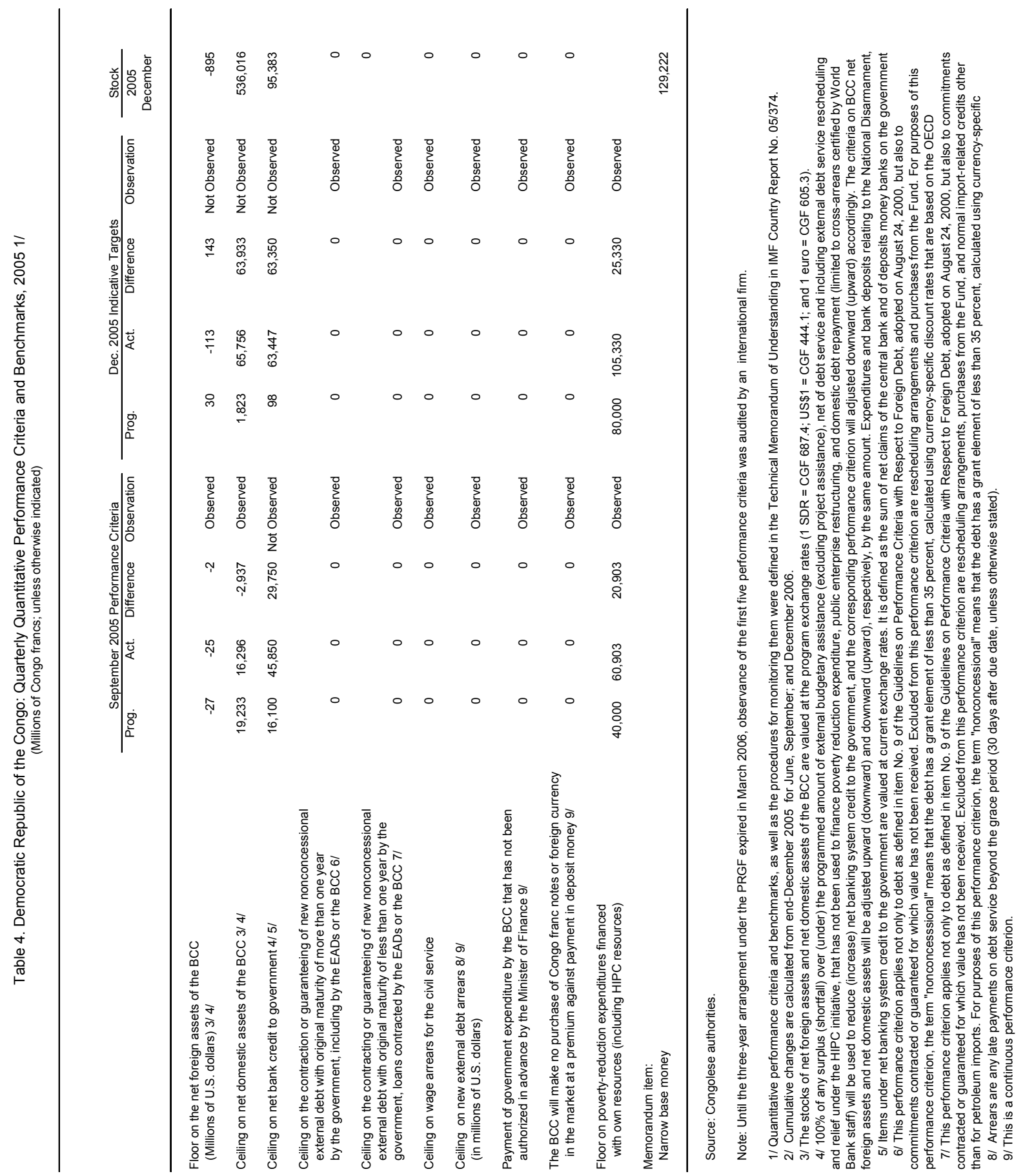




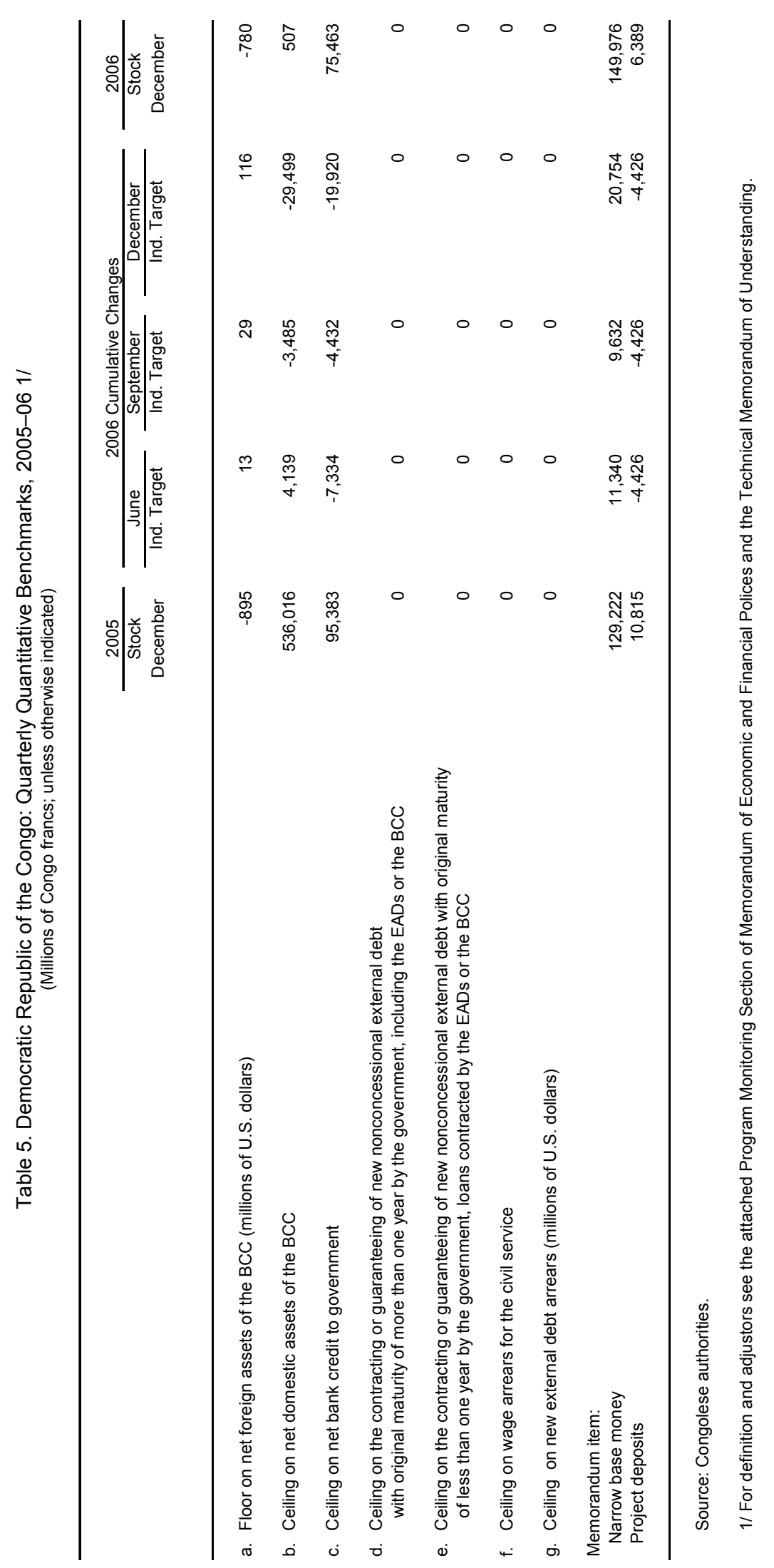




\title{
APPENDIX I \\ ATTACHMENT II \\ TRANSLATED FROM FRENCH
}

\author{
Democractic Republic Of The Congo \\ TECHNICAL MEMORANDUM OF UNDERSTANDING
}

Kinshasa, June 29, 2006

1. This memorandum covers the agreements on monitoring implementation of the staff monitored program (SMP), for the period April-December 2006, by the staff of International Monetary Fund (IMF). It establishes the information to be reported and the deadlines for its submission to the IMF staff for program monitoring. It defines the quantitative benchmarks, as well as the structural benchmarks presented in the Memorandum on Economic and Financial Policies (MEFP) of the government of the Democratic Republic of the Congo (DRC), which is attached to the letter of June 29, 2006 to the Managing Director of the International Monetary Fund.

\section{A. Monitoring Program Implementation}

2. Implementation of the program covering the period April, 1 to December, 31, 2006 will be monitored on the basis of the benchmarks described in Tables 2 and 5 of the MEFP of June 29, 2006.

\section{B. Definition of Quantitative Benchmarks}

3. The quantitative benchmarks described in Table 5 of the MEFP are as follows:

a. floor on net foreign assets of the central bank (BCC);

b. ceiling on net domestic assets of the BCC;

c. ceiling on net bank credit to the government;

d. ceiling on new nonconcessional external loans contracted or guaranteed by the central government, including those contracted by the decentralized autonomous entities (EADs) or the BCC, with maturities of more than one year, except borrowing for debt rescheduling purposes, and IMF credit;

e. ceiling on new nonconcessional external loans contracted or guaranteed by the central government, and loans contracted by the EADs and the BCC, with maturities of one year or less, except borrowing for debt rescheduling purposes, IMF credit, and normal import credits (suppliers' credits), excluding imports of petroleum products;

f. $\quad$ ceiling on wage arrears (see paragraph 9 for definition); 
The following benchmarks will be monitored on a continuous basis:

g. the government shall not accumulate external payments arrears on debt service, including loans for which a debt rescheduling agreement has been concluded with the government's creditors, or on any new borrowing. Arrears are any late payments on the debt service beyond the grace period (30 days after due date, unless otherwise stated).

\section{Definitions}

4. Net foreign assets of the $\mathbf{B C C}$ are defined as the difference between the BCC's gross foreign assets and all its foreign liabilities, as shown in the "BCC Balance Sheet" prepared by the BCC. For purposes of the relevant benchmarks, the net foreign assets will be valued in U.S. dollars at the program exchange rates: US $\$ 1=$ CGF 444.1; SDR $1=$ CGF 687.4; and EUR 1 = CGF 605.3.

5. The net domestic assets of the BCC are equal to the sum of the following line items, as they appear in the BCC balance sheet, except for 'other items, net' which are valued in $\mathrm{CGF}$ at the current exchange rate. For the relevant benchmark, domestic assets will be adjusted for the effect of the CGF/U.S. dollar exchange rate variation from the programmed rate on net foreign assets and foreign exchange deposits and provisions for imports, and on 'other items, net' (revaluation). (See point 4. above):

- $\quad$ net claims on the government;

- $\quad$ claims on nonfinancial public enterprises;

- $\quad$ claims on the nonfinancial private sector;

- $\quad$ claims on banks;

- $\quad$ claims on other banking and nonbank institutions; and

- "other items, net," defined as other assets minus other liabilities (including capital and revaluation accounts, and liquidity management instruments (Billets de Trésorerie) purchased by deposit money banks and the public).

6. Net banking system credit to the government is defined as the sum of net claims of the central bank and of deposit money banks on the government, as defined in the "Integrated Monetary Survey" prepared by the BCC (excluding deposits linked to project-related assistance; see paragraph 7 above), plus the BCC's net cash deficit. Items under net bank credit to the government are valued at the current exchange rates.

7. The end-June and end-September 2006 benchmarks on net bank credit to the government and the BCC's net domestic (foreign) assets will be adjusted downward (upward) for one hundred percent of any shortfall from the programmed amount of net external debt service payments made, and for fifty percent of any surplus over the 
programmed amount of external budgetary assistance (excluding project assistance), including external debt rescheduling or relief.

The end-December 2006 targets on net bank credit to the government and the BCC's net domestic (foreign) assets will be adjusted upward (downward), up to a maximum of CF15 billion, for one hundred percent of any shortfall from the programmed amount in external budgetary assistance net of debt service payments including external debt service rescheduling and relief.

Conversely, the end-December 2006 targets on net bank credit to the government, and on the BCC's net domestic (foreign) assets will be adjusted downward (upward) for fifty percent of any surplus over the programmed amount in external budgetary assistance net of debt service payments including external debt service rescheduling and relief.

For the computation of the above adjustors, expenditures and bank deposits relating to the National Disarmament, Demobilization, and Reintegration Program (DDR), elections, and domestic debt are considered projects. Their financing through external assistance are therefore excluded from external budgetary assistance. The adjustors do not apply to HIPC resources, which will be deposited in a special account in the BCC. The adjustors will be in US dollars for the floor on the BCC's net foreign assets, and in Congo Francs (using the current exchange rate) for the ceilings on net bank credit to the government and on the BCC's net domestic assets.

8. Debt relief under the HIPC initiative is defined: (a) for bilateral and commercial creditors, as the difference between the debt service due after possible stock treatment under the Naples terms at end-2002 and post-HIPC service due (excluding relief beyond the HIPC Initiative), including only arrangements that have been signed or are to be signed within the year, and; (b) for multilateral creditors, as the difference between the service due after consolidation of arrears and post-HIPC service. Any debt relief for the DRC under the HIPC initiative will be credited to the HIPC account (account of the Treasury General Account in the $\mathrm{BCC}$ ) in the same month that said relief is realized (i.e., on the date the service on the original debt owed is due, but cancelled under the HIPC initiative). The procedure for using the HIPC account is set out in the IMF staff report on the HIPC decision point (IMF Country Report No. 03/267).

9. Wages comprise all remuneration, payable to government employees during the month in question (active and retired civil servants, military personnel, national police and security personnel, diplomats, members of political and traditional institutions), including regular compensation and bonuses, but excluding nonrecurring compensation. Under the program, wage arrears for a given month are defined as wages unpaid at the end of the following month. For remuneration paid in the provinces, the arrears are measured on the basis of the balances of the accounts of the provincial delegated payment authorization officers (ODs) in the Treasury General Account at the BCC. 
10. The definition of the external debt can be found in the Fund's Decision 6230(79/140), paragraph 9, revised on August 24, 2000 (Annex I).

11. The grant element of borrowing will be calculated on the basis of currency-specific rates based on the OECD commercial interest reference rates (CIRR) on the date the contract is signed, as specified in the Annex. A loan is defined as concessional if, on the date the contract is signed, the ratio of the present value of the loan, calculated on the basis of the reference interest rate, to its nominal value is less than 65 percent (i.e., including a grant element of at least 35 percent).

12. Base money is defined as the sum of the following:

- $\quad$ currency in circulation (in and outside banks);

- deposits of banks with the BCC;

- deposits of public enterprises with the BCC;

- $\quad$ deposits of private enterprises and individuals with the BCC; and

- deposits of other financial institutions, other than deposit money banks, with the $\mathrm{BCC}$, and;

- $\quad$ foreign exchange deposits and provisions for imports at the BCC.

Note: "Base money" excludes all central bank bills issued by the BCC, which are counted as other BCC liabilities, and which are included in BCC net domestic assets. Narrow base money is defined as base money less foreign exchange deposits and provisions for imports with the BCC. Narrow base money is valued at the actual exchange rate (see paragraph 4 above).

13. The following concepts are used in the letter of intent and the Memorandum on Economic and Financial Policies:

a. Budget: annual law authorizing the government's financial operations. Transfers to the provinces are included, but the provinces' own revenues are not covered. The social security system is not consolidated in the budget;

b. Supplementary budgets (budgets annexes): Budgets of agencies and entities similar to administrative public enterprises that are funded primarily by government transfers (rétrocessions) and subsidies;

c. Extrabudgetary accounts: accounts receiving government revenue not tracked by the Treasury Management and Payment Authorization Directorate, or not included in the supplementary budgets. These accounts are consolidated with those regularly monitored by the Treasury Management and Payment Authorization Directorate; 
d. Poverty-reduction expenditure: "pro-poor" spending as defined in the new nomenclature on the basis of the priorities set forth in the I-PRSP and detailed in Annex II.

\section{Structural Benchmarks}

14. The structural benchmarks are described in Table 2 of the Memorandum on Economic and Financial Policies.

\section{Reporting}

15. The authorities will forward to the IMF's African Department, preferably by e-mail or fax, the data and information needed to monitor program implementation. These data and information must be duly reconciled so as to ensure their internal consistency. Following are the data or documents to be submitted:

\section{Exchange system}

The following information is to be submitted with a time lag of one day.

a. Volume of purchases and sales of foreign exchange on the interbank market, by the BCC (interventions), by commercial banks, and by exchange bureaus;

b. Average CGF/U.S. dollar reference exchange rate of the BCC (indicative rate);

c. Average CGF/U.S. dollar exchange rate on the interbank market;

d. Average CGF/U.S. dollar exchange rate offered by commercial banks to their customers; and,

e. Average CGF/U.S. dollar exchange rate used by exchange bureaus.

\section{Banking system}

The following monthly information is to be submitted no later than four weeks after the end of each month, unless otherwise stated.

a. Integrated monetary survey, with a breakdown into domestic currency and foreign currency;

b. BCC balance sheet, with a breakdown into domestic currency and foreign currency;

c. BCC operating account, and implementation of the BCC's cash flow plan;

d. Consolidated balance sheet of deposit money banks, with a breakdown into domestic currency and foreign currency;

e. Volume of purchases and sales of BCC bills (interventions) by commercial banks and by the public (every week);

f. Net banking system credit to the government;

g. $\quad$ Structure of nominal and real interest rates of deposit money banks;

h. $\quad$ Reserves (voluntary and required) of deposit money banks;

i. $\quad$ Structure of BCC interest rates; and

j. Structure of rates for central bank bills. 


\section{Public sector}

\section{Unless otherwise indicated, the following information is to be submitted not later than four weeks after the end of each month.}

a. Implementation of Treasury cash flow plan (TOFE);

b. Budget tracking statements (états de suivi budgétaire, ESB reconciled with the TOFE, detailing expenditures according to economic, administrative, and functional classifications, and by province - see Annex III);

c. Table: 'Monitoring of Wage Arrears' with following details: wage bill, wages paid from the Treasury General Account broken down by employees categories, region (Kinshasa/provinces), and status (active/retired);

d. Civil service pay scale (if changed);

e. Government revenue broken down by collecting agencies, individual taxes, and sources of nontax revenue; tax and non tax payments by oil producing companies;

f. Public sector domestic debt, by category of creditor (commercial banks, private entities, etc.): collect and report data on domestic public debt as soon as they are available;

h. Payments and invoiced amounts on centralized expenditures.

i. Posting on the websites of the ministry of finance and the ministry of budget of summaries of the monthly cash flow plan and spending plan for the coming three months and the monthly outturn for each "service".

\section{Real sector}

Report within four weeks of the end of each month (unless otherwise indicated) the following indicators of recent economic developments: the consumer price index (on a weekly basis), monthly merchandise exports (value and volume) of crude oil, copper, cobalt, zinc, industrial and hand cut diamonds; imports (value and volume) by main categories (raw materials, intermediate goods, consumer goods, capital goods, and petroleum products); and output indicators for the manufacturing, mining, and services sectors, published in the BCC's monthly reports on economic activity. 


\section{External debt}

The following monthly information is to be provided four weeks after the end of each month.

a. Disbursements of external loans and grants, including those associated with new loans contracted (on a monthly basis, with a lag of three weeks);

b. Monthly debt service payments (interest and principal) classified by creditor, made in foreign currency (source: the Public Debt Management Office-OGEDEP, and the BCC) and in CGF (source: DTO);

c. Monthly external debt service obligations before and after debt relief; arrears on external debt obligations (stock and new arrears) broken down by principal and interest, and classified by creditors (to be provided monthly by OGEDEP), and;

d. Copies of (i) the new loans contracted, including those contracted by the EADs; and (ii) debt rescheduling agreements with the Paris Club, non-Paris Club bilateral creditors, commercial creditors, and multilateral creditors, within four weeks of the signing of such agreements.

\section{Miscellaneous}

A progress report on implementation of the structural reforms will be submitted to Fund staff each month. In addition, information on the legal and regulatory environment as it affects business (new decrees, circulars, and laws) and price policy, as well as the official gazette, will also be reported to Fund staff.

\section{Jean-Claude Masangu Mulongo}

Governor

Central Bank of the Congo
M.F. Muamba Tshishimbi

Minister of Budget
Marco Banguli

Minister of Finance

Kinshasa, June 29, 2006 


\section{APPENDIX I \\ ATTACHMENT II \\ ANNEX I \\ TRANSLATED FROM FRENCH}

\section{Definition of External Debt}

1. The definitions of "debt" and "concessional borrowing" for the purposes of this memorandum of understanding are as follows:

a. As set out in Point 9 of the Guidelines on Benchmark with Respect to Foreign Borrowing adopted by the IMF's Executive Board on August 24, 2000, debt is understood to mean a current, that is, not contingent, liability created under a contractual agreement through the provision of value in the form of assets (including currency) or services, and which requires the obligor to make one or more payments in the form of assets (including currency) or services at some future points in time; these payments will discharge the principal and/or interest liabilities incurred under the contract. Debt can take a number of forms, the primary ones being as follows: (i) loans, that is, advances of money to the obligor by the lender on the basis of an undertaking that the obligor will repay the funds in the future (including deposits, bonds, debentures, commercial loans, and buyers' credits) and temporary exchanges of assets that are equivalent to fully collateralized loans, under which the obligor is required to repay the funds, and usually pay interest, by repurchasing the collateral from the buyer in the future (such as repurchase agreements and official swap arrangements); (ii) suppliers' credits, that is, contracts where the supplier permits the obligor to defer payment until some time after the date on which the goods are delivered or services are provided; and (iii) leases, that is, arrangements under which property is provided that the lessee has the right to use for one or more specified period(s) of time, which are usually shorter than the total expected service life of the property, while the lessor retains the title to the property. For the purpose of this guideline, the debt is the present value (at the inception of the lease) of all lease payments expected to be made during the period of the arrangement, excluding those payments that cover the operation, repair, or maintenance of the property. Under the definition of debt set out above, arrears, penalties, and judicially awarded damages arising from failure to make payment under a contractual obligation that constitutes debt are debt. Failure to make payment on an obligation that is not considered debt under this definition (e.g., payment on delivery) will not give rise to debt.

b. A loan is considered concessional if, on the date the contract is signed, the ratio of the present value of the loan, based on the reference interest rates, to the nominal value of the loan is less than 65 percent (i.e., a grant element exceeding 35 percent). The reference interest rates used in this assessment are the commercial interest reference 
rates (CIRRs) established by the Organization for Economic Cooperation and Development (OECD). For debts with a maturity exceeding 15 years, the ten-year reference interest rate published by the OECD is used to calculate the grant element. For shorter maturities, the six-month market reference rate is used. 


\section{APPENDIX I \\ ATTACHMENT II \\ ANNEX II \\ TRANSLATED FROM FRENCH}

\section{Definitions of Poverty Reducing Spending}

\section{The concept}

Poverty reducing spending comprises all outlays by the government aimed at improving the living standards and well-being of the people, based on the priorities established in the Interim Poverty Reduction Strategy Paper (PRSP) and the final PRSP.

\section{Benchmarks}

Poverty reducing spending defined in the budget on the basis of the classification for the general functions of government. reducing:

Spending on the following functions and sub-functions are considered to be poverty

02 Defense

02. 600 Reintegration of demobilized soldiers

03 Security

03. 300 The judiciary and court system

03. 320 Courts

04 Economic Affairs

04. 200 Agriculture, forestry, hunting, fisheries, and rural development

04. 210 Agriculture, livestock

04. 220 Forestry

04. 230 Hunting and fishing

04. 240 Rural development

04. 300 Fuel and electricity

04. 350 Electricity (in the provinces)

04. 500 Transport

04. 510 Roads

04. 511 National roads

04. 512 Provincial roads

04. 513 Farm roads

04. 514 Urban roads

04. 520 Sea, river and lake transport

04. 530 Railroads

05 Protection of the Environment and of Flora and Fauna 
05. 100 Household waste management

05. 200 Sewage and sanitation

05. 300 Pollution control

05. 400 Protection of fauna and flora

\section{Public Housing and Facilities}

06. 100 Housing development

06. 200 Equipment of community facilities, regional and urban development

06. 300 Water supply

06. 400 Street lighting

$07 \quad$ Health

07. 100 Medicines, prostheses, and medical equipment and supplies

07. 110 Medicines and pharmaceuticals

07. 120 Light medical equipment

07. 130 Prostheses and medical supplies

07. 200 Local medical services (outpatient care)

07. 210 General medical services

07. 220 Specialized medical services

07. 230 Dental services

07. 240 Paramedical services (including traditional medicine, physiotherapy, and labs)

07. 300 Hospital services

07. 310 General hospitals

07. 320 Specialized hospitals

07. 330 Maternity clinics and dispensaries

07. 400 Public health services

07. 410 Preventive care

07. 420 Treatment

07. 430 Vaccination

07. 440 Polio vaccination campaign

07. 432 Other vaccination campaigns

07. 440 Epidemic control programs

07. 441 AIDS program

07. 442 Malaria program

07. 443 Tuberculosis program

07. 444 Programs for other epidemics

07. 500 Research and development in the field of health

60 Other health issues (includes mainly wages of health professionals)

09 Education

09. 100 Pre-school and elementary education

09. 110 Pre-school education 
09. 120 Elementary education

09. 200 Secondary education

09. 210 General secondary education

09. 220 Technical and professional secondary education

09. 400 Higher education

09. 410 General higher education

09. 420 Technical, professional, and administrative higher education (only equipment and repair of infrastructure, Articles 71.20, 72.10, 73.10, 74.10, 75.20, 81.10, and 82.10).

500 Other education

09. 510 General and professional continuing training for adults

09. 800 Other educational affairs (Wages of educational employees)

10 Social Security and Welfare

10. 100 Diseases and disabilities

10. 110 Diseases

10. 120 Disabilities

10. 200 Aging

10. 210 Social security for the elderly

10. 400 Family and children

10. 500 Unemployment programs

10. 600 Housing and housing conditions

10. 700 Social exclusion programs 


\section{APPENDIX I \\ ATTACHMENT II \\ ANNEX III \\ TRANSLATED FROM FRENCH}

\section{Budget Tracking Statements}

\section{Report 1: Main budget tracking report with economic classification of expenditure (ESB principal). Monthly.}

This report provides data on expenditures at the level of the four steps in the expenditure procedure (commitment, verification, payment order, payment), on the one hand, and by economic classes, on the other, and cumulatively from the start of the fiscal year (2004

Revised Classification).

The last column of the main budget tracking statement is the "Balances Outstanding" (Restes à Payer comptables, RAP) column, which is the difference between payment orders and actual payments by the BCC.

\section{Report 2: Budget tracking report with administrative classification. Monthly.}

Based on the main statement, this document will present expenditures according to administrative classification.

\section{Report 3: Budget tracking report, by “Major Government Functions.” Monthly.}

This document will present expenditure by major government function.

\section{Report 4: Budget tracking report, by source of financing. Monthly.}

This report provides data on total expenditure according to sources of financing, economic classification and cumulatively from the start of the fiscal year.

\section{Report 5: Budget tracking report with administrative and economic classifications. Monthly.}

Based on the main statement and the one with the administrative classification, this document will present expenditure according to administrative and economic classification.

\section{Report 6: Budget tracking statement, Poverty-Reducing Expenditure by economic} classification. Monthly.

Based on Report 1, poverty reducing expenditure broken down according to economic classification. 
Report 7: Budget tracking statement, Poverty-Reducing Expenditure by administrative classification. Monthly.

Based on Report 1, poverty reducing expenditure broken down according to administrative classification.

Report 8: Budget tracking statement, Poverty-Reducing Expenditure by government functions. Monthly.

Based on Report 3, poverty reducing expenditure broken down according to main functions of government.

Report 9: Budget tracking report, Poverty-Reducing Expenditure by source of financing. Monthly.

This report provides data on total expenditure according to sources of financing, economic classification and cumulatively from the start of the fiscal year. It should identify poverty reducing expenditure financed through domestic sources and HIPC savings.

Report 10: Budget tracking report, Poverty-Reducing Expenditure by geographical distribution. Monthly source of financing. Monthly.

This report provides data on poverty reducing expenditure broken down by provinces, economic classification, and cumulatively from the start of the fiscal year. It should identify poverty reducing expenditure executed in Kinshasa and in individual provinces. 


\section{APPENDIX II \\ Democratic Republic OF THE CONGo \\ RELATIONS WITH THE FUND \\ (As of May 31, 2006)}

I. Membership Status: Joined: September 28, 1963; Article VIII

II. General Resources Account:

Quota

Fund holdings of currency

\section{SDR Department:}

Net cumulative allocation

Holdings

IV. Outstanding Purchases and Loans:

Poverty Reduction and Growth Facility (PRGF) arrangement
SDR Million

533.00

533.00

SDR Million

86.31

0.09

SDR Million

553.47
In Percent of Quota 100.00 100.00

In Percent of Allocation 100.00 0.11

In Percent of Quota 103.84

\section{Latest Financial Arrangements:}

\begin{tabular}{lllcc} 
Type & \multicolumn{1}{c}{$\begin{array}{c}\text { Approval } \\
\text { Date }\end{array}$} & $\begin{array}{c}\text { Expiration } \\
\text { Date }\end{array}$ & $\begin{array}{c}\text { Amount Approved } \\
\text { (SDR Million) }\end{array}$ & $\begin{array}{c}\text { Amount Drawn } \\
\text { (SDR Million) }\end{array}$ \\
PRGF & June 12, 2002 & March 31, 2006 & 580.00 & 553.47 \\
Stand-By & June 9, 1989 & June 8, 1990 & 116.40 & 75.00 \\
SAF & May 15, 1987 & May 14, 1990 & 203.70 & 145.50
\end{tabular}

\section{Projected Payments to Fund}

(SDR million; based on existing use of resources and present holdings of SDRs):

\begin{tabular}{lrrrrr} 
& \multicolumn{5}{c}{ Forthcoming } \\
\cline { 2 - 6 } & $\mathbf{2 0 0 6}$ & $\mathbf{2 0 0 7}$ & $\mathbf{2 0 0 8}$ & $\mathbf{2 0 0 9}$ & $\mathbf{2 0 1 0}$ \\
Principal & 0.00 & 42.00 & 86.67 & 97.34 & 105.35 \\
Charges/interest & 4.33 & 5.86 & 5.54 & 5.07 & 4.55 \\
Total & 4.33 & 47.86 & 92.21 & 102.41 & 109.91
\end{tabular}




\section{Implementation of HIPC Initiative:}

I. Commitment of HIPC assistance

Enhanced

Decision point date

Framework

Assistance committed by all creditors (millions U.S. dollars)

July 2003

Of which: IMF assistance (millions U.S. dollars)

$6,311.00$

318.90

(SDR equivalent millions)

228.30

Completion point date

Floating

II. Disbursement of IMF assistance (millions SDRs)

Assistance disbursed to the member

Interim assistance

Completion point balance

Additional disbursement of interest income

Total disbursements

VIII. Implementation of MDRI Assistance: Not Applicable

\section{Exchange Rate Arrangement:}

The Democratic Republic of the Congo's (DRC) currency is the Congo franc, which, since May 26, 2001, has been freely floating. On May 31, 2006, the rate was US\$1=Congo francs 449.0

Effective February 10, 2003, the DRC accepted the obligations of Article VIII, Sections $2(a), 3$, and 4, of the Fund's Articles of Agreement. However, the DRC maintains measures that give rise to one restriction and one multiple currency practice (MCP) subject to Fund approval under Article VIII of the Fund's Articles of Agreement. The exchange restriction involves an outstanding net debit position vis-à-vis other contracting members under the inoperative regional payments agreement with the Economic Community of the Great Lakes Countries (CEPGL). The multiple currency practice involves a fixed exchange rate set on a quarterly basis applying to transactions through the bilateral payments agreement (BPA) with Zimbabwe.

\section{Last Article IV Consultation:}

(a) Consultations with the Democratic Republic of the Congo are held in accordance with the provisions of the decision on consultation cycles approved on July 15, 2002.

(b) The last Article IV consultation was concluded by the Executive Board on August 19, 2005. 


\section{Safeguards Assessment}

A safeguards assessment of the BCC was completed on January 3, 2003. The assessment concluded that substantial risks of misreporting or misuse may exist due to vulnerabilities in the external audit mechanism, financial reporting framework, and system internal controls. Staff findings, proposed recommendations under program conditionality, and other recommendations are reported in Country Report No. 03/161. Implementation of the measures by the BCC continues to be monitored by staff.

\section{Technical Assistance:}

\begin{tabular}{|c|c|c|c|}
\hline Subject & Department & Staff Member & Date \\
\hline Monetary statistics & STA & Mr. Papadacci & Jun. 29-Jul. 12, 2000 \\
\hline & & Ms. Tanase & \\
\hline Exchange rate system & MFD & Mr. Bussers & May 9-14, 2001 \\
\hline & & & Jul. 1-7, 2001 \\
\hline Article VIII obligations & MFD/LEG & Ms. De Boeck & Oct. $21-29,2002$ \\
\hline & & Mr. Leimone & \\
\hline \multirow{14}{*}{$\begin{array}{l}\text { Capacity-building } \\
\text { assistance to the central } \\
\text { bank }\end{array}$} & \multirow[t]{14}{*}{ MFD } & Mr. Laurens & Oct. $12-26,2001$ \\
\hline & & Mr. Sarr & \\
\hline & & Mr. Akitoby & \\
\hline & & Mr. Paul & \\
\hline & & Mr. Bussers & \\
\hline & & Mr. Tavernier & Feb. 1-14, 2002 \\
\hline & & & Aug. 8-Sep. 20, 2002 \\
\hline & & Mr. Paul & Feb. 1-7, 2002 \\
\hline & & Mr. Beaumé & Jul. 15-25, 2002 \\
\hline & & Mr. Tavernier & Oct. 10-22, 2004 \\
\hline & & Mr. Normand & \\
\hline & & Mr. Saffre & Nov. 8-12, 2004 \\
\hline & & Mr. Cotier & Mar. 03-Apr. 07, 2006 \\
\hline & & Mr. Le Clerc & \\
\hline \multirow{6}{*}{$\begin{array}{l}\text { Monetary policy/ } \\
\text { foreign exchange } \\
\text { management, bank } \\
\text { supervision and budget }\end{array}$} & \multirow[t]{6}{*}{ MFD } & Mr. Laurens & \multirow[t]{6}{*}{ Oct. 29-Nov. 12, 2002} \\
\hline & & Mr. Sarr & \\
\hline & & Mr. Paul & \\
\hline & & Mr. Beaumé & \\
\hline & & Mr. Régnard & \\
\hline & & Mr. Tavernier & \\
\hline
\end{tabular}




\begin{tabular}{|c|c|c|c|}
\hline $\begin{array}{l}\text { Action plan for } \\
\text { developing the } \\
\text { financial system in the } \\
\text { DRC }\end{array}$ & MFD & $\begin{array}{l}\text { Mr. Laurens } \\
\text { Mr. Fonteyne } \\
\text { Ms. Nkhata } \\
\text { Mr. Saffré } \\
\text { Mr. Paul } \\
\text { Mr. Regnard } \\
\text { Mr. Vandecan } \\
\text { Mr. Pauwels }\end{array}$ & Nov. 5-15, 2003 \\
\hline $\begin{array}{l}\text { Assistance to the } \\
\text { central bank on } \\
\text { currency, monetary and } \\
\text { foreign exchange } \\
\text { operations, and } \\
\text { strengthening of } \\
\text { capacity in AML/CFT }\end{array}$ & MFD & $\begin{array}{l}\text { Mr. Nascimiento } \\
\text { Mr. Vandecan } \\
\text { Mr. Lecinq } \\
\text { Mr. Van de Velde } \\
\text { Mr. Hotte }\end{array}$ & Nov. 30 - Dec. 14, 2004 \\
\hline $\begin{array}{l}\text { Monetary and Foreign } \\
\text { Exchange Operations, } \\
\text { Currency in } \\
\text { Circulation, and } \\
\text { Banking Supervision }\end{array}$ & MFD & $\begin{array}{l}\text { Mr. Nascimiento } \\
\text { Mr. Hartley } \\
\text { Mr. Van de Velde } \\
\text { Mr. Lecinq } \\
\text { Mr. Cotier }\end{array}$ & Nov. 29-Dec 13, 2005 \\
\hline Currency in circulation & MFD & Mr. Van de Velde & $\begin{array}{l}\text { Mar. 28-Apr. 10, } 2005 \\
\text { March 03-Apr. 07, } 2006\end{array}$ \\
\hline Monetary operations & MFD & Mr. Lecinq & Sept. 05-16, 2005 \\
\hline $\begin{array}{l}\text { Bank supervision and } \\
\text { regulation }\end{array}$ & MFD & Mr. Regnard & Nov. 22-Dec. 05, 2004 \\
\hline Internal audit & MFD & $\begin{array}{l}\text { Mr. Normand } \\
\text { Mr. Tavernier }\end{array}$ & Mar. 26-Apr. 8, 2006 \\
\hline $\begin{array}{l}\text { Legislative and } \\
\text { supervisory AML/CFT } \\
\text { framework }\end{array}$ & MFD & $\begin{array}{l}\text { Ms. Smith } \\
\text { Mr. Mathias } \\
\text { Mr. Beekarry }\end{array}$ & Jan. 23-27, 2006 \\
\hline $\begin{array}{l}\text { Tax administration/ } \\
\text { policy }\end{array}$ & FAD & $\begin{array}{l}\text { Mr. Corfmat } \\
\text { Mr. Fossat }\end{array}$ & May $11-21,2001$ \\
\hline Tax policy & FAD & $\begin{array}{l}\text { Ms. Geourjon } \\
\text { Mr. Laporte } \\
\text { Mr. Schneider }\end{array}$ & Nov. 19-Dec. 3, 2002 \\
\hline \multirow[t]{2}{*}{$\begin{array}{l}\text { Expenditure } \\
\text { management }\end{array}$} & FAD & $\begin{array}{l}\text { Mr. Schiller } \\
\text { Mr. Fournel } \\
\text { Mr. Barrier }\end{array}$ & Aug. 4-18, 2001 \\
\hline & & $\begin{array}{l}\text { Mr. Bouley } \\
\text { Mr. Calcoen }\end{array}$ & Aug. 31-Sep. 10, 2002 \\
\hline
\end{tabular}




\begin{tabular}{|c|c|c|c|}
\hline & & $\begin{array}{l}\text { Mr. Bouley } \\
\text { Mr. Lepage } \\
\text { Mr. Kwant }\end{array}$ & Nov. 11-25, 2003 \\
\hline \multirow[t]{4}{*}{$\begin{array}{l}\text { Revenue } \\
\text { administration }\end{array}$} & \multirow[t]{4}{*}{ FAD } & $\begin{array}{l}\text { Mr. Benon } \\
\text { Mr. Jolibert } \\
\text { Mr. Montagnard-Rentier }\end{array}$ & Dec. 3-17, 2002 \\
\hline & & $\begin{array}{l}\text { Mr. Benon } \\
\text { Mr. Lesprit }\end{array}$ & Jul. 13-26, 2003 \\
\hline & & $\begin{array}{l}\text { Mr. Benon } \\
\text { Mr. Montagnat-Rentier } \\
\text { Mr. Lesprit } \\
\text { Mr. Boilil } \\
\text { Mr. Vandenberghe }\end{array}$ & $\begin{array}{l}\text { Sep. } 16-20,2003 \\
\text { Oct. } 26-\text { Nov. } 9,2004\end{array}$ \\
\hline & & Mr. Boilil & Apr 10-30, 2006 \\
\hline Fiscal Decentralization & FAD & $\begin{array}{l}\text { Mr. Seade } \\
\text { Mr. Brosio } \\
\text { Mr. Catalan } \\
\text { Mr. Hartley } \\
\text { Mr. Mati } \\
\text { Mr. Raouya } \\
\text { Ms. Kubota (World } \\
\text { Bank) } \\
\text { Mr. Vaillancourt (World } \\
\text { Bank) }\end{array}$ & Jul. 15-31, 2004 \\
\hline $\begin{array}{l}\text { Real sector and } \\
\text { government finance } \\
\text { statistics }\end{array}$ & STA & $\begin{array}{l}\text { Mr. Marie } \\
\text { Mr. Gorter }\end{array}$ & Jun. 4-13, 2001 \\
\hline $\begin{array}{l}\text { Government finance } \\
\text { statistics }\end{array}$ & STA & Mr. Maiga & $\begin{array}{l}\text { Mar. 19-Apr. 13, } 2002 \\
\text { Sep.4-25, } 2002\end{array}$ \\
\hline Multisector statistics & STA & $\begin{array}{l}\text { Ms. Fisher } \\
\text { Mr. Tanase } \\
\text { Ms. Noukovska } \\
\text { Mr. Fiévet } \\
\text { Mr. Maiga } \\
\text { Mr. Ntungwanayo (World } \\
\text { Bank) }\end{array}$ & Oct. 8-21, 2003 \\
\hline $\begin{array}{l}\text { Tax administration } \\
\text { Central Africa } \\
\text { Regional Expert }\end{array}$ & FAD & Mr. Fossat & Sept. 6, 2005 \\
\hline
\end{tabular}




\section{Long-term Resident Experts:}

Expenditure management

Customs administration

Tax administration

Advisor to Governor of BCC

Capacity building of $\mathrm{BCC}$

Statistics
Mr. Catalan

Mr. Nguenang

Mr. Bremeersch

Mr. Schlotterbeck

Mr. Fossat

Mr. Raouya

Mr. d'Ambrières

Mr. Nyssens

Mr. d'Ambrières

Mr. Pauwels

Mr. Métreau

Mr. Gbossa
Jan. 2002-Jun. 2004

Since Sep. 2004

Jan. 2002-Jan. 2004

Jan. 2002-Mar. 2003

Sept 2005

Sep. 2003-Apr. 2005

Apr. 2002-Dec. 2003

Jan. 2004-

Feb. 252006

Since Sep. 2004

Oct. 2004-

Since Dec. 122005

IV. Resident Representative: Mr. Maret assumed his duties as Resident Representative on November 30, 2005. 


\section{Introduction}

22. This annex first underscores the importance that the government of the Democratic Republic of the Congo (DRC) attaches to effective partnership with international development partners and foreign investors. It then summarizes the strategy and activities of the World Bank Group (IDA, IFC, and MIGA) in the DRC. It concludes with a description of areas of specific collaboration between the World Bank Group and the International Monetary Fund.

\section{Partnership in the DRC's development strategy}

23. The DRC is in the final weeks of preparing for the first democratic elections in decades, which are scheduled to start in July 2006. These elections will mark the end of the transition period that began when the country emerged from the conflict (1997-2001). The conflict caused estimated 3.8 million deaths, most of which were due to disease and hunger, as well as extensive destruction of infrastructure and the collapse of institutions.

24. President Joseph Kabila has made it a priority to reestablish relations with international financial institutions when he took office in January 2001. The IMF responded with a staff-monitored program (SMP), starting in June 1, 2001, and IDA approved a Transitional Support Strategy (TSS) in July 2001. The progress exhibited by the Government supporting peace and good performance under the programs with the Bretton Woods Institutions led to substantial financial reengagement by multilateral and bilateral partners. As a result of the DRC's engagement with the international community, the Paris Club granted significant relief on bilateral debt at its September 2002 meeting. Additionally, the efforts of the DRC to work towards an improved political, economic, and social environment were recognized by the IMF and IDA Executive Directors in July 2003 when the decision point under the enhanced HIPC Initiative was reached. Government efforts to implement change and improve the political, economic and social environment have continued and are supported by development partners.

\section{The PRSP has been finalized and submitted to the Government for formal}

adoption after extensive participatory consultations at grassroots level as well as with the international development partners. The adoption is expected to take place by end June 2006. Since the adoption of the I-PRSP in June 2002, the first and second annual Preparation Status

\footnotetext{
3 This document was approved by the Africa Region Operations Committee on June 21, 2006. Questions should be addressed to Keiko Kubota (202-473-6836) or Heather Milkiewicz (202-473-3632) at the World Bank.
} 
Reports and related Joint Staff Assessments (JSAs) were produced, and discussed by the IMF and IDA Boards in July 2003 and 2004, respectively.

\section{Bank Group strategy and operations (IDA, IFC, and MIGA) ${ }^{4}$}

26. Overall strategy. On July 31, 2001, the Executive Directors of IDA endorsed a Transitional Support Strategy to map out Bank financial and nonfinancial support to the DRC for the following 12-24 months, which included the Emergency Early Recovery Project (EERP), the Economic Recovery Credit (ERC), the Private Sector Development and Competitiveness Project (PSDC), and the Emergency Multi-Sector Rehabilitation and Reconstruction Project (EMRRP), as described hereunder. The second TSS was approved by the Board on February 26, 2004 and covers the period 2004-06. A Country Assistance Strategy (CAS) covering the period of 2006-09 is being prepared in order to be presented to the Executive Directors of IDA depending on consultations with the new government to be elected.

27. The 2004 TSS sets forth the scope and configuration of future support during the 2004-06 period. The TSS accompanies the government's efforts during the transition period, with a particular focus on (i) social stability and security, (ii) high and shared growth, (iii) governance and institutional strengthening, and (iv) social development.

\section{Existing support}

\section{IDA}

IDA provides support through trust funds, development policy support, and investment operations. Operational details are summarized in Table 1.

\section{(i) Trust funds}

The trust funds that are currently active target the areas of stabilization and recovery, demobilization, capacity building, support for the PRSP, forest governance, and arrears clearance. Together these trust funds make up over US\$1.3 billion.

\section{(ii) Grants and credits}

\section{Development policy support}

28. Since the re-engagement, IDA has provided three policy reform operations - the Economic Recovery Credit (ERC), the Post-Reunification Economic Recovery Credit (PRERC), and the Transitional Support to Economic Recovery Operation (TSERO). The ERC, approved in June 2002 in the amount of SDR 360.4 million (US\$450 million

\footnotetext{
${ }^{4}$ The Bank reopened a country office in Kinshasa, and a country manager assumed his duties in January 2002.
} 
equivalent at the time of negotiations), provided support to the arrears-clearance process, to a voluntary departures program at GECAMINES (the national copper mining company), to forestry sector reforms, and to key public expenditure reforms. The operation was implemented satisfactorily and closed in June 2003. The PRERC, approved in February 2004 in the amount of SDR 135.2 million (US\$200 million equivalent at the time of negotiations), provided support to a civil service retirement program, a domestic debt settlement program, and utilities billing and payment reforms. Conditions for tranche release involved the adoption of adequate reform strategies plus fiduciary arrangements to ensure that funds reach intended recipients. The operation was implemented satisfactorily and closed in December 2005. The focus of the TSERO, approved in December 2005 in the amount of SDR 62.1 million (US\$90 million equivalent at the time of negotiations), was on the continuation of improvements to the budget process, strengthening the execution of pro-poor spending, implementation of civil service management reforms, the continuation of support for reforms in natural resource sectors, and increased transparency on the revenue side. Unlike the previous two operations, which had multiple thematic tranches, TSERO was a single tranche programmatic operation. It disbursed fully in December 2005, and is scheduled to close on December 31, 2006

\section{Traditional investment operations}

29. The IDA provides support through investment operations in the DRC in the areas of (i) infrastructure rehabilitation - the Emergency Early Recovery Project (EERP), the Emergency Multi-Sector Rehabilitation and Reconstruction Project (EMRRP), the Emergency Economic and Social Reunification Support Project, and the Southern Africa Power Market Project (SAPM); (ii) demobilization and reintegration - the Emergency Demobilization and Reintegration Project (EDRP); (iii) emergency support to living conditions- Emergency Living Conditions Improvement Support Project; (iv) private sector development - the Private Sector Development and Competitiveness Project (PSDC); (v) debt reduction- Debt Buy Back Operation; (vi) social development-HIV/AIDS (MultiSectoral HIV/AIDS Program), and the Health Sector Rehabilitation Support Project. Implementation of projects to date is broadly satisfactory, except for the EDRP and Emergency Social Action Project.

30. With respect to infrastructure rehabilitation, the Emergency Early Recovery Project (EERP) rehabilitated the Matadi-Kinshasa road. It has been fully disbursed. As of March 2006, close to 100 percent of the funds (both credit and grant) for the Emergency Multi-Sector Rehabilitation and Reconstruction Project (EMRRP, which largely finances basic infrastructure rehabilitation) had been committed and more than 50 percent disbursed. An additional financing to the EMRRP has been approved in December 2005 and will also support infrastructure rehabilitation. The Emergency Economic and Social Reunification Support Project focuses on infrastructure rehabilitation in the reunified provinces, and 46 percent of the credit has been disbursed. The SAPM will strengthen the capacity of the Coordination Center of the Southern Africa Power Pool (SAPP) to promote and manage electricity trade in the region; remove transmission bottlenecks; and connect member countries to the regional grid, which will foster economic growth. The DRC component of 
the SAPP is the largest and will enable the DRC to supply electricity to neighboring countries. It was approved in November 2003, and implementation is in progress.

31. With respect to demobilization and reintegration, the Emergency Demobilization and Reintegration Project provides support to the demobilization and reintegration of 150,000 ex-combatants country-wide. The project was developed within the framework of the Multi-Country Demobilization and Reintegration Program (MDRP) for the Great Lakes region, a regional partnership initiative comprising seven governments and over 40 donor and multilateral agencies. The MDRP partners endorsed a US\$100 million MDRP trust fund grant (cofinancing) to complement US\$100 million in IDA grant financing. Collaboration in the field between the Bank, other donors and multilateral agencies is proceeding well, and IDA is paying particular attention to the deepening of cooperation. Demobilization started in February 2005. Sixty-eight thousand former combatants have been demobilized, a secure system for paying ex-combatants a transitional safety net allowance has been established and 20 reintegration centers have been opened. Demobilization is expected to be completed by mid-2006. While the project continues to advance, there are important overall management problems and concerns remain about project financial management and procurement. Full reintegration of the former combatants is also a challenge.

32. The Emergency Social Action Project, approved by the World Bank in August 2004, will channel funds to poor communities to finance socio-economic infrastructure determined by the communities. This project became effective in July 2005 but has not advanced as expected. Project management has been poor, with significant staff turnover. The scholarship component has been well-implemented, but the primary project activity of financing community proposals has not progressed.

33. With respect to private sector development, the PSDC is helping to reform inefficient public enterprises and improving the investment climate to attract private investment, which would contribute to economic growth. The Debt Buy Back Operation, approved in September 2005, will contribute to clearing of the remaining arrears owed by the Government.

34. With respect to HIV/AIDS, the Multi-Sectoral HIV/AIDS project, approved in March 2004, is helping to mitigate the negative impact of HIV/AIDS on the socioeconomic development of the DRC. By end December 2005, more than 200 subprojects and micro projects have been funded for US\$14 million, of which US\$9.2 million have been disbursed. Among the project's accomplishments to date are the following: 82 percent of the 50,000 people targeted annually are using Voluntary Counseling and Treatment (VCT) centers; 90 percent of those targeted annually are treated for STDs according to acceptable standards, and 3 percent of orphans (out of 8 percent targeted) have been assisted for tuition and schooling. However, in terms of the quality medical treatment given to those living with HIV/AIDS, only 5 percent of the objective has been realized. Thus far, Kinshasa (the capital) has benefited from the majority of the funding. However, the remaining 6 provinces that are not yet covered will benefit from the program before the end of July 2006. On another note, the project experienced procurement problems but is in the process of correcting them. 
Another project which contributed to reforms in the area of HIV/AIDS is the Emergency Early Recovery Project (EERP). The EERP improved the security of the blood supply, which will aid in preventing the transmission of HIV/AIDS. 


\section{Table 1. Summary of World Bank Financial Assistance}

\begin{tabular}{|c|c|c|c|c|}
\hline Credit Name & Amount & Date approved & Theme & Status \\
\hline $\begin{array}{l}\text { Emergency Early } \\
\text { Recovery Project } \\
\text { (EERP) }\end{array}$ & $\begin{array}{l}\text { US\$50 million } \\
\text { IDA grant }\end{array}$ & Jul. 31, 2001 & $\begin{array}{l}\text { Economic reforms, a pilot } \\
\text { community-driven development } \\
\text { initiative, rehabilitation of the } \\
\text { Kinshasa-Matadi road, and } \\
\text { HIV/AIDS activities }\end{array}$ & $\begin{array}{l}\text { Completed and } \\
\text { fully disbursed }\end{array}$ \\
\hline $\begin{array}{l}\text { Economic Recovery } \\
\text { Credit (ERC) }\end{array}$ & $\begin{array}{l}\text { US } \$ 450 \\
\text { million }\end{array}$ & Jun. 13, 2002 & $\begin{array}{l}\text { Arrears clearance, budgetary/foreign } \\
\text { exchange support, and forestry and } \\
\text { mining sector reforms }\end{array}$ & $\begin{array}{l}\text { Completed and } \\
\text { fully disbursed }\end{array}$ \\
\hline $\begin{array}{l}\text { Emergency Multi- } \\
\text { Sector Rehabilitation } \\
\text { and Reconstruction } \\
\text { Project (EMRRP) }\end{array}$ & $\begin{array}{l}\text { US\$454 } \\
\text { million, o/w } \\
\text { US\$44 million } \\
\text { grant. } \\
\text { Supplementary } \\
\text { funding of } \\
\text { \$125 million } \\
\text { was approved } \\
\text { on Dec. 8, } \\
2005 \text {. }\end{array}$ & Aug. 6, 2002 & $\begin{array}{l}\text { Reconstruction and rehabilitation of } \\
\text { critical infrastructure, increase in social } \\
\text { service delivery, institutional capacity } \\
\text { strengthening, development of sectoral } \\
\text { strategies }\end{array}$ & $\begin{array}{l}\text { As of March } \\
2006 \text {, close to } \\
100 \text { percent of } \\
\text { the initial funds } \\
\text { (credit and } \\
\text { grants) had been } \\
\text { committed and } \\
\text { more than } \\
50 \text { percent } \\
\text { disbursed. }\end{array}$ \\
\hline $\begin{array}{l}\text { Private Sector } \\
\text { Development and } \\
\text { Competitiveness } \\
\text { Project (PSDC) }\end{array}$ & $\begin{array}{l}\text { US\$120 } \\
\text { million }\end{array}$ & Jul. 29, 2003 & $\begin{array}{l}\text { Increase competitiveness by improving } \\
\text { the investment climate, support reform } \\
\text { of public enterprises, stimulate } \\
\text { economic diversification in Katanga, } \\
\text { and provide job search support for the } \\
\text { unemployed }\end{array}$ & Ongoing \\
\hline $\begin{array}{l}\text { Emergency Economic } \\
\text { and Social } \\
\text { Reunification Support } \\
\text { Project }\end{array}$ & $\begin{array}{l}\text { US } \$ 214 \\
\text { million, o/w } \\
\text { US } \$ 164 \\
\text { million grant }\end{array}$ & Sep. 11, 2003 & $\begin{array}{l}\text { Finance emergency rehabilitation } \\
\text { activities (large infrastructure, urban } \\
\text { rehabilitation, community } \\
\text { development, in particular to the } \\
\text { reunified provinces) }\end{array}$ & $\begin{array}{l}47 \text { percent of } \\
\text { credit has been } \\
\text { disbursed }\end{array}$ \\
\hline $\begin{array}{l}\text { Southern Africa Power } \\
\text { Market Project } \\
\text { (SAPM) }\end{array}$ & $\begin{array}{l}\text { US\$178.6 } \\
\text { million }\end{array}$ & Nov. 11, 2003 & $\begin{array}{l}\text { Restore capacity to deliver electric } \\
\text { power to SAPP }\end{array}$ & Ongoing \\
\hline $\begin{array}{l}\text { Post-Reunification } \\
\text { Economic Recovery } \\
\text { Credit (PRERC) }\end{array}$ & $\begin{array}{l}\text { US\$200 } \\
\text { million }\end{array}$ & Feb. 26, 2004 & $\begin{array}{l}\text { Support to civil service retirement } \\
\text { program, settlement of government } \\
\text { debt arrears to private creditors, and } \\
\text { reform of utilities billing and payment }\end{array}$ & $\begin{array}{l}\text { Credit fully } \\
\text { disbursed }\end{array}$ \\
\hline $\begin{array}{l}\text { Multi-sectoral } \\
\text { HIV/AIDS program } \\
\text { (MAP) }\end{array}$ & $\begin{array}{l}\text { US\$102 } \\
\text { million }\end{array}$ & Mar. 26, 2004 & $\begin{array}{l}\text { Mitigate the negative impact of the } \\
\text { HIV/AIDS epidemic on the } \\
\text { socioeconomic development of the } \\
\text { DRC through prevention of } \\
\text { transmission, support, and care for } \\
\text { persons living with the virus (PLV) }\end{array}$ & $\begin{array}{l}\text { Ongoing. } \\
\text { Project effective } \\
\text { since Oct. } 8 \text {, } \\
2004\end{array}$ \\
\hline $\begin{array}{l}\text { Emergency } \\
\text { Demobilization and } \\
\text { Reintegration Project }\end{array}$ & $\begin{array}{l}\text { US\$100 } \\
\text { million IDA } \\
\text { grant; plus } \\
\text { US } \$ 100 \\
\text { million in } \\
\text { counterpart } \\
\text { (MDRP) funds } \\
\text { (grants) }\end{array}$ & May 25, 2004 & $\begin{array}{l}\text { Demobilize } 150,000 \text { ex-combatants } \\
\text { country-wide and help them transition } \\
\text { to civilian life, help increase social and } \\
\text { economic expenditures, and lower } \\
\text { defense expenditures }\end{array}$ & $\begin{array}{l}71 \text { percent of the } \\
\text { IDA grant and } \\
32 \text { percent of the } \\
\text { MDRP grant } \\
\text { were disbursed } \\
\text { by end-March } \\
2006\end{array}$ \\
\hline
\end{tabular}




\begin{tabular}{|c|c|c|c|c|}
\hline Credit Name & Amount & Date approved & Theme & Status \\
\hline $\begin{array}{l}\text { Emergency Social } \\
\text { Action Project }\end{array}$ & $\begin{array}{l}\text { US\$60 million } \\
\text { IDA grant }\end{array}$ & Aug. 6, 2004 & $\begin{array}{l}\text { Improve access of the poor to social } \\
\text { and economic services and increase the } \\
\text { availability and management of } \\
\text { development resources at the } \\
\text { community level }\end{array}$ & Ongoing \\
\hline $\begin{array}{l}\text { Emergency Living } \\
\text { Conditions } \\
\text { Improvement Support } \\
\text { Project }\end{array}$ & $\begin{array}{l}\text { US\$ } 82 \\
\text { million IDA } \\
\text { grant }\end{array}$ & May 26,2005 & $\begin{array}{l}\text { The Project aims to assist the } \\
\text { Government in responding to multi- } \\
\text { sector emergency needs in urban areas } \\
\text { - including large cities (other than } \\
\text { Kinshasa), as well as key medium and } \\
\text { small urban centers }\end{array}$ & Ongoing \\
\hline $\begin{array}{l}\text { Health Sector } \\
\text { Rehabilitation Support } \\
\text { Project }\end{array}$ & $\begin{array}{l}\text { US\$150 } \\
\text { million IDA } \\
\text { grant }\end{array}$ & Sept. 1, 2005 & $\begin{array}{l}\text { This project will ensure that the target } \\
\text { population of selected health zones has } \\
\text { access to and use of a well-defined } \\
\text { package of quality essential health } \\
\text { services. In particular, this project will } \\
\text { support the implementation of the } \\
\text { Ministry of Health's (MOH) Package } \\
\text { of Essential Health Services (EHS) in } \\
83 \text { selected Health Zones in the } \\
\text { provinces of Equateur }\end{array}$ & Ongoing \\
\hline $\begin{array}{l}\text { Debt Buy Back } \\
\text { Operation }\end{array}$ & $\begin{array}{l}\text { US\$ } 900 \\
\text { thousand } \\
\text { IDA grant }\end{array}$ & Sept. 8, 2005 & $\begin{array}{l}\text { This project will address the } \\
\text { commercial debt of the DRC and } \\
\text { facilitate the provisions of the debt } \\
\text { relief by the remaining commercial } \\
\text { creditors }\end{array}$ & Ongoing \\
\hline $\begin{array}{l}\text { Transitional Support to } \\
\text { Economic Recovery } \\
\text { Operation (TSERO) }\end{array}$ & $\begin{array}{l}\text { US\$90 million } \\
\text { IDA grant }\end{array}$ & Dec. 8,2005 & $\begin{array}{l}\text { Support for the improvement of the } \\
\text { budget and revenue-collecting process, } \\
\text { execution of pro-poor spending, } \\
\text { natural resource sector management, } \\
\text { and implementation of civil service } \\
\text { management reforms }\end{array}$ & $\begin{array}{l}\text { Completed and } \\
\text { fully disbursed }\end{array}$ \\
\hline
\end{tabular}




\section{Nonlending activities}

35. For several years prior to and during the first TSS (2001), IDA assistance also emphasized nonlending activities and advisory services, including through trust funds and grants, to improve the understanding of the socioeconomic context, rebuild the knowledge base to support policy dialogue and design effective poverty-reduction strategies. FY03 activities included a Public Expenditure Review, which focused on the overall structure of expenditures; specific issues in the health, education, and transport sectors; evaluation of the DRC's ability to monitor execution of poverty-related expenditures; strengthening of public expenditure management systems. Also in FY03 support was given to the PRSP process, especially the consultation and participatory diagnostic processes, an update of the poverty profile, and a Debt Sustainability Analysis for the HIPC Initiative program, undertaken in collaboration with the IMF. In FY04, a Country Procurement Assessment Review was undertaken. The final version of the CSR-Health was sent to the Government on May 13, 2005. An Education Sector Country Status Report was completed in October 2004 and served as the basis for discussion regarding the preparation of a strategy for the education sector. Activities in FY05 included the HIPC/AAP pro-poor expenditure-tracking exercise, an Institutional Governance Review (focusing on service delivery issues, distributed to the Government in June 2005), Poverty Assessment (first draft sent to the Government in June 2005), a Forestry Sector Review (in draft), an Agricultural Sector Review and the Risk and Vulnerability Assessment. In FY06, the Country Financial Accountability Assessment (CFAA) was undertaken, and was officially delivered to the Government and the donor community in Kinshasa. A workshop was also organized for Government delegates, the Reform Committee, Parliament, Cour des Comptes and several donors to validate the conclusions and action plan of the report. The Agricultural Sector Review was completed in FY06.

\section{International Finance Corporation}

36. IFC supported the cellular telephone operator Celtel with a US\$7 million loan in 2002 and a further US\$20 million in 2003. Currently, IFC is conducting a needs assessment of local small and medium enterprises to help determine potential future interventions. IFC has made a US\$4.9 million equity investment for a 7.5 percent equity stake in Kingamyambo Musonoi Tailings SARL (KMT), which is among the first mining projects to be developed under the new Mining Code. This project has recently completed the necessary feasibility, environmental and social studies. IFC was actively involved in the preparation, review and approval of the environmental and social studies. The next step will be to raise the financing of the project and begin construction planned for end-2006 or early 2007. The total cost of the project is estimated at US $\$ 400$ million. Timing and project scope could change as a result of the recent takeover of KMT's parent company (Adastra Minerals) by First Quantum Minerals. In the financial sector, IFC plans to assist banks operating in the DRC with trade financing facilities in order to help establish routine trade finance operations. IFC will work closely with the Bank in the context of the PSDC project to help implement specific sectoral initiatives and key investment projects. Considerable effort has already been spent in resolving outstanding disputes in the DRC, two of which, UTEXAFRICA, a textile business, 
and the Grand Hotel du Congo (formerly Inter-Continental) were settled in 2002 and 2005, respectively. A settlement is still being sought for the last dispute remaining on the books, SOTEXKI, a textile business in Kisangani.

\section{MIGA}

37. MIGA can now issue guarantees for projects because the DRC has paid its initial capital subscription (the DRC has been a member of the agency since 2003). Several projects are under consideration, notably in the mining sector. On May 3, 2005, MIGA approved its first project in DRC for a US\$13.3 million in guarantee coverage for the Dikulushi copper and silver mine. The guarantees approved provide coverage for investments and loans by Anvil Mining Ltd. of Canada and RMB International (Dublin) Limited of Ireland to Anvil Mining Congo, SAR of the DRC, against the risks of transfer restriction, expropriation, breach of contract and war and civil disturbance. The project scope involves open pit mining of copper and silver ores to produce a concentrate, which will be transported to smelters in South Africa and Namibia for further processing. The project was also the first extractive industries project to be considered and approved by the Board of the World Bank Group, which followed the conclusion of the Bank-sponsored Extractive Industries Review in August 2004.

\section{Bank-Fund collaboration in specific areas}

38. In addition to its direct assistance to the DRC, the Bank also supports policy reforms in close collaboration with the Fund in a number of areas: donor coordination, public finance management, decentralization, public enterprise reform, financial sector reform, governance and anticorruption, and the PRSP process. Table 2 details areas of collaboration and areas where the either the Bank or the Fund lead. 
Table 2. Summary of Bank-Fund Collaboration

\begin{tabular}{|c|c|c|c|}
\hline Thematic area & Areas of collaboration & Bank & Fund \\
\hline $\begin{array}{l}\text { Donor } \\
\text { coordination }\end{array}$ & $\begin{array}{l}\text { Promotion of international } \\
\text { partnership activities }\end{array}$ & $\begin{array}{l}\text { Preparation of Consultative Groups, and } \\
\text { monitoring of individual aid flows. }\end{array}$ & $\begin{array}{l}\text { Integration and } \\
\text { monitoring of financial } \\
\text { partnership activities in } \\
\text { the macroeconomic } \\
\text { framework }\end{array}$ \\
\hline \multirow[t]{2}{*}{$\begin{array}{l}\text { Public finance } \\
\text { management }\end{array}$} & $\begin{array}{l}\text { Ensuring compatibility of } \\
\text { information systems; public } \\
\text { finance management; public } \\
\text { expenditure tracking (HIPC- } \\
\text { AAP exercises) and } \\
\text { monitoring; poverty-related } \\
\text { spending, monitoring, and } \\
\text { evaluation }\end{array}$ & $\begin{array}{l}\text { Policy reform support, particularly in the area } \\
\text { of information systems for budget preparation, } \\
\text { budget execution, fiscal reporting, and public } \\
\text { debt recording/management. } \\
\text { Preparing public expenditure reviews and } \\
\text { CFAA. }\end{array}$ & $\begin{array}{l}\text { Reforming and } \\
\text { modernizing revenue and } \\
\text { expenditure management } \\
\text { systems. Technical } \\
\text { assistance on reform of } \\
\text { public sector accounting } \\
\text { system }\end{array}$ \\
\hline & $\begin{array}{l}\text { Preparing proposed reforms of } \\
\text { customs and internal indirect } \\
\text { taxes, corporate taxation; and } \\
\text { reforming taxation in mining } \\
\text { and forestry sectors }\end{array}$ & $\begin{array}{l}\text { Financing technical assistance in the area of } \\
\text { training and the structure of corporate taxation; } \\
\text { conducting in-depth analysis of forestry } \\
\text { taxation systems. }\end{array}$ & $\begin{array}{l}\text { Technical assistance for } \\
\text { improving tax } \\
\text { administration }\end{array}$ \\
\hline Decentralization & Fiscal decentralization & $\begin{array}{l}\text { Technical assistance and advice on the } \\
\text { decentralization of public finances and service } \\
\text { delivery; improving the tracking of public } \\
\text { expenditures. }\end{array}$ & $\begin{array}{l}\text { Technical assistance for } \\
\text { improving tax } \\
\text { administration and } \\
\text { expenditure management }\end{array}$ \\
\hline $\begin{array}{l}\text { Public enterprise } \\
\text { reform }\end{array}$ & $\begin{array}{l}\text { Collaboration on the public } \\
\text { finance aspects of these } \\
\text { reforms (e.g., cross arrears, } \\
\text { corporate tax regimes) }\end{array}$ & $\begin{array}{l}\text { Preparation and execution of public enterprise } \\
\text { sector reform ; and improving the legal, } \\
\text { regulatory, judicial, and fiscal environment for } \\
\text { private sector development. }\end{array}$ & -- \\
\hline $\begin{array}{l}\text { Financial sector } \\
\text { reform }\end{array}$ & -- & Restructuring of commercial banks. & $\begin{array}{l}\text { Monetary policy issues } \\
\text { and central bank } \\
\text { restructuring }\end{array}$ \\
\hline \multirow[t]{2}{*}{$\begin{array}{l}\text { Governance and } \\
\text { anticorruption }\end{array}$} & $\begin{array}{l}\text { Improving the codes of } \\
\text { conduct for public servants, as } \\
\text { well as strengthening } \\
\text { anticorruption and anti- } \\
\text { money-laundering efforts }\end{array}$ & $\begin{array}{l}\text { Aiding in preparation of workshops and } \\
\text { seminars on the formulation of an overall } \\
\text { governance and anticorruption strategy; } \\
\text { providing advice and comments on legislation } \\
\text { as needed. }\end{array}$ & $\begin{array}{l}\text { Providing advice and } \\
\text { comments on legislation } \\
\text { as needed }\end{array}$ \\
\hline & & $\begin{array}{l}\text { Financing technical assistance for a } \\
\text { comprehensive procurement reform: } \\
\text { preparation of a Country Procurement } \\
\text { Assessment Review, production of a new } \\
\text { procurement code, the implementation of a } \\
\text { system of procurement follow-up, and } \\
\text { reorganization of public entities in charge of } \\
\text { the procurement process. }\end{array}$ & \\
\hline PRSP process & $\begin{array}{l}\text { Joint assessments of I-PRSP } \\
\text { and PSRs }\end{array}$ & $\begin{array}{l}\text { Aiding in preparation of consultations and } \\
\text { workshops, coordination of donor funding, } \\
\text { recruitment of national experts for Permanent } \\
\text { Secretariat, and the National Poverty Survey. }\end{array}$ & $\begin{array}{l}\text { Implementation of } \\
\text { macroeconomic } \\
\text { framework in PRSP }\end{array}$ \\
\hline
\end{tabular}


Table 3. The Democratic Republic of Congo: Financial Relations with the World Bank Group-Statement of Loans and Credits (in U.S. dollars), as of May 31, 2006

\begin{tabular}{lrrrr}
\hline & IBRD & IDA & IDA Grant & Total \\
\hline Original principal & $330,000,000$ & $2,773,794,622$ & $967,000,000$ & $4,070,794,622$ \\
Cancellations & $28,484,478$ & $259,852,029$ & 0 & $288,336,507$ \\
Disbursed & $301,515,522$ & $2,213,573,135$ & $368,293,838$ & $2,883,382,495$ \\
Undisbursed & 0 & $502,466,976$ & $616,160,433$ & $1,118,627,409$ \\
Repaid & $247,045,475$ & $250,014,155$ & 0 & $497,059,631$ \\
Due & 0 & $2,177,433,100$ & 0 & $2,177,433,100$ \\
Exchange adjustment & 0 & 0 & 0 & \\
Borrower's obligation & 0 & $2,177,433,100$ & 0 & $2,177,433,100$ \\
\hline
\end{tabular}




\title{
Democratic Republic of the Congo: Progress Status of Triggers for Reaching the Floating Completion Point
}

\author{
(As of June 2006)
}

\begin{abstract}
Trigger
1. PRSP

Completion of a full PRSP through a participatory process and its implementation for one year, duly documented in the DRC's annual progress report, and confirmed as satisfactory by a joint staff assessment (JSA).

\section{Macroeconomic stability}

Continued maintenance of macroeconomic stability after reaching the decision point, as evidenced by satisfactory performance under a program supported by an arrangement under the IMF's PRGF.
\end{abstract}

\section{Use of budgetary savings}

Use of budgetary savings resulting from enhanced HIPC Initiative-related debt service relief during the interim period for poverty-related expenditures in accordance with the I-PRSP, with supporting documentation satisfactory to the staffs of IDA and the IMF.

\section{Public expenditure management}

(a) Implementation of a modernized budget-execution system, providing information from commitment to payment, and allowing for the monitoring of arrears; (b) adoption and implementation of a double-entry government accounting system and a new chart of accounts; and (c) production of quarterly budget execution reports using economic, administrative and functional classifications.

\section{Status}

The latest version of the Poverty Reduction Strategy Paper (PRSP) was circulated to development partners in June 2006 and is expected to be adopted toward mid-2006.

Following the incorporation of the comments and assistance received from the development partners, the final PRSP is expected to be adopted by government by end-June 2006 .

The DRC was successful in restoring macroeconomic balances under the PRGF-supported program. Real GDP growth turned positive, reaching 6.5 percent in 2005 , inflation fell sharply, and the exchange rate became more stable. The economic situation has remained so far stable in 2006.

The use of budgetary savings from the enhanced HIPC Initiative has improved. The government continues its efforts to use HIPC savings to finance spending in the areas of health and education. An external audit of the HIPC account is planned for 2006.

(a) The budget-execution system is improving. However, additional efforts are needed to ensure timely and comprehensive transmission of information to relevant parties;

(b) The double-entry system is being implemented. The staff have been trained, equipment purchased, and software developed;

(c) A new classification has been adopted. The budgetary reports are produced but not within the 30 days after the closing of the month. 


\section{Trigger}

\section{Governance and service delivery in priority sectors}

(a) Completion of a budget-tracking exercise on health, education, rural development and infrastructure expenditure, consisting of (i) monitoring the execution of poverty-related public expenditure; (ii) evaluation by user groups of the quality of related public services, and (iii) evaluation by service-

(b) adoption and implementation of a new procurement code and key implementing decrees.

\section{Social and rural sectors}

Adoption of sectoral development strategies and related implementation plans for health, education and rural development, which are satisfactory to IDA. providers of constraints to effective provision; and

\section{Status}

(a) The new budget classification allows tracking of poverty reducing and social expenditures. However, the budget reports produced so far do not fully incorporate expenditures using external resources, which makes accurate evaluation difficult.

b) The Steering Committee has adopted the draft Procurement Code in April 2006; the revised version will be submitted for adoption to the Council of Ministers. The consultants are drafting the SBDs, guides and manuals as well as the decrees creating the new institutions to be established when the Code is promulgated

An initial draft of the Government Report on Health and Poverty (RESP) was finalized in December 2004. In the field of education, the government finalized, in September 2004, the Status Report on the National Education System (RESEN), which assesses the sector's constraints, and adopted a draft action plan for the Education for All (EFA) Initiative. An agriculture sector review was completed in May 2006, and will serve as an input to the rural development strategy.

\section{Debt Management}

Installation and full activation of a computerized debtrecording system, covering public and publiclyguaranteed debt that can (a) produce monthly debtservice projections, and incorporate actual disbursement and debt-service payment execution data; (b) produce advance monthly debt-service projections that will be published quarterly; and (c) support the centralization of debt information into a single center.
A public debt management software (SYGADE) was installed, and recording of the external debt outstanding is complete. The recorded data is now being checked for consistency. Training of the relevant staff on debt management is on-going. The implementation program is projected to be completed by end- 2006 . 